\title{
Mixed Convection over an Inclined Wavy Surface in a Nanofluid Saturated Non-Darcy Porous Medium with Radiation Effect
}

\author{
Darbhasayanam Srinivasacharya and Poshala Vijay Kumar \\ Department of Mathematics, National Institute of Technology, Warangal, Telangana 506004 , India \\ Correspondence should be addressed to Darbhasayanam Srinivasacharya; dsrinivasacharya@yahoo.com
}

Received 3 July 2015; Revised 17 July 2015; Accepted 12 August 2015

Academic Editor: Alírio Rodrigues

Copyright ( 2015 D. Srinivasacharya and P. Vijay Kumar. This is an open access article distributed under the Creative Commons Attribution License, which permits unrestricted use, distribution, and reproduction in any medium, provided the original work is properly cited.

\begin{abstract}
The problem of mixed convection in a nanofluid along an inclined wavy surface embedded in a non-Darcy porous medium with radiation effect is analyzed. Coordinate transformation is employed to transform the complex wavy surface to a smooth surface. The governing equations are transformed into a set of ordinary differential equations using the appropriate similarity transformation and then solved using the successive linearization method. The present results are compared with previously published work and are found to be in very good agreement. The effect of pertinent parameters on the nondimensional velocity, temperature, nanoparticle volume fraction, heat, and nanoparticle mass transfer rates is studied and presented graphically.
\end{abstract}

\section{Introduction}

The research on heat transfer in nanofluids is receiving much attention from the past several years due to its unique characteristics in thermal engineering fields and broad range of applications such as microelectronics, microfluidics, solidstate lighting, transportation, biomedical, and manufacturing. To enhance the thermal conductivity and viscosity of the base fluid, colloidal suspensions of nanometer sized particles (of dimensions about $1-100 \mathrm{~nm}$ ) are made to form nanofluid [1]. Choi et al. [2] showed that the addition of a small amount (less than $1 \%$ by volume) of nanoparticles to conventional heat transfer liquids increased the thermal conductivity of the fluid. The nanofluids are advantageous in the sense that they are stable and have an acceptable viscosity and better wetting, spreading, and dispersion properties. A detailed review of the literature on nanofluids and applications of nanofluids can be found in the book by Das et al. [3] and collection of papers by Kakaç and Pramuanjaroenkij [4] and Puliti et al. [5]. These reviews discuss in detail the work done on convective transport in nanofluids.

Numerous models and methods have been proposed by different authors to study convective flows of nanofluids. The computational studies reported in this area include two main approaches: (1) a two-phase model, in which both liquid and solid heat transfer behaviors are solved in the flow fields [6, 7], and (2) a single-phase model, in which solid particles are considered to behave as fluids, because the nanoparticles are easily fluidized [8-10]. Buongiorno [11] showed that the high heat transfer coefficients in nanofluids cannot be explained satisfactorily by thermal dispersion phenomenon or increase in turbulence intensity promoted by the presence of nanoparticles or nanoparticle rotation as suggested in the literature. He noted that the nanoparticle absolute velocity can be viewed as the sum of the base fluid velocity and a relative velocity (which he calls the slip velocity). He developed a mathematical model to capture the nanoparticle/base fluid slip by treating nanofluid as a two-component mixture with Brownian diffusion and thermophoresis as the important factors in the convective transport process in a nanofluid. Kuznetsov and Nield [12] used the Buongiorno [11] model to study the natural convective flow of a nanofluid over a vertical plate. Buongiorno [11] model is the basis of the present study.

Convective transport in porous media has been widely studied in recent years due to its wide range of applications in mechanical, chemical, and civil engineering. A review of convective heat transfer in porous medium is presented in the book by Nield and Bejan [13]. Considerable work has been 
reported in the literature focusing on the problem of mixed convection of nanofluid along an inclined surface embedded in porous medium. Ahmad and Pop [14] studied the steady mixed convection boundary layer flow past a vertical flat plate embedded in a porous medium filled with nanofluids using different types of nanoparticles and the model they used for the nanofluid incorporates only the nanoparticle volume fraction parameter. Chamkha and Aly [15] obtained numerical solution of steady natural convection boundary layer flow of a nanofluid consisting of a pure fluid with nanoparticles along a permeable vertical plate in the presence of magnetic field, heat generation or absorption, and suction or injection effects. Rosca et al. [16] numerically studied the problem of steady mixed convection boundary layer flow over an impermeable horizontal flat plate embedded in a porous medium saturated by a nanofluid. Rana et al. [17] obtained the numerical solution for steady mixed convection boundary layer flow of an incompressible nanofluid along an inclined plate in a porous medium. Kamali and Binesh [18] numerically studied the effects of nanoparticles size on convective heat transfer characteristics of metal oxide nanofluids considering thermal conductivity and viscosity of nanofluid as a function of nanoparticles size, volume fraction, and Brownian motion.

Radiation effects on convection gained importance in the context of many industrial applications involving high temperatures such as gas turbines, nuclear power plant, and various propulsion engines for aircraft, missiles, satellites, and space technology. Chen et al. [19] studied the effects of thermal radiation on laminar forced and free convection along a wavy surface. Chamkha et al. [20] presented the nonsimilar solution of steady mixed convection of a nanofluid in the presence of thermal radiation. Hady et al. [21] studied the effect of radiation on viscous flow of a nanofluid and heat transfer over a nonlinearly stretching sheet. Abdel-Rahman [22] numerically investigated the problem of thermal radiation and unsteady MHD flow of a nanofluid in stretching porous medium.

The prediction of heat and mass transfer from irregular surface has great importance in engineering applications. In many practical situations surfaces are roughened in order to enhance the rate of heat transfer. The presence of roughened surface not only alters the flow field but also alters the heat and mass transfer characteristics. Natural convection from wavy surfaces is used for transferring heat in several heat transfer devices, such as flat plate condensers in refrigerators, flat plate solar collectors, and cooling of electrical and nuclear components. Extensive studies of natural convection heat and mass transfer of a vertical wavy surface under boundary layer approximation have been undertaken by several authors. Mahdy and Ahmed [23] have analyzed laminar free convection over a vertical wavy surface embedded in a porous medium saturated with a nanofluid. Ahmed and Abd El-Aziz [24] studied the effect of local thermal nonequilibrium on unsteady heat transfer by natural convection of a nanofluid over a vertical wavy surface. On the other hand, convection along inclined surfaces and bluff bodies has been receiving the attention because of many industrial applications in areas such as electroplating, chemical processing of heavy metals,

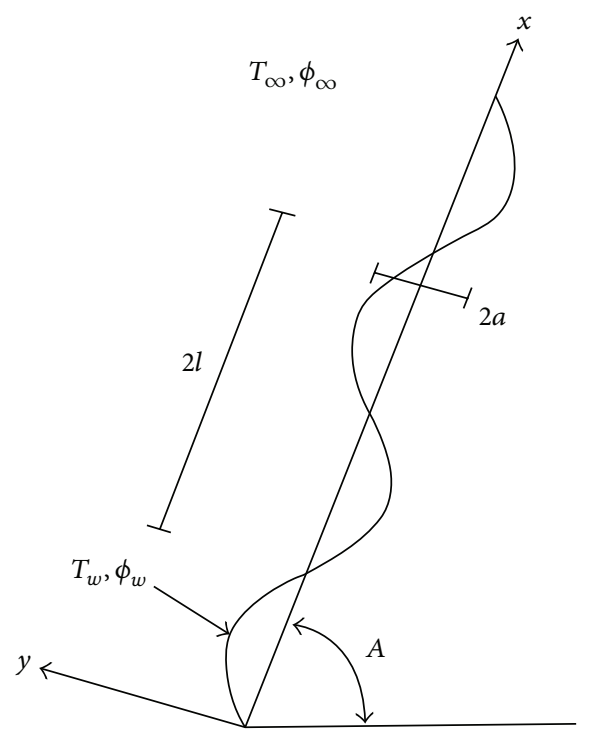

FIgURE 1: Physical model.

and ash or scrubber waste treatment. Ingham et al. [25] studied the natural convection from a semi-infinite flat plate inclined at a small angle to the horizontal in a saturated porous medium. Cheng $[26,27]$ studied the double diffusive natural convection along an inclined wavy surface in a porous medium and bidisperse porous medium.

In this study, we aimed at exploring the effects of radiation and the angle of inclination of a wavy surface on mixed convection in a non-Darcy porous medium saturated with a nanofluid.

\section{Mathematical Formulation}

Consider the steady laminar incompressible two-dimensional boundary layer mixed convection flow along a semiinfinite inclined wavy surface embedded in a nanofluid saturated non-Darcy porous medium. The coordinate system is shown in Figure 1. The wavy surface is described by

$$
y=\delta(x)=a \sin \left(\frac{\pi x}{l}\right)
$$

where $a$ is the amplitude of the wavy surface and $2 l$ is the characteristic length of the wavy surface. The wavy surface is maintained at uniform wall temperature $T_{w}$ and nanoparticle concentration $\phi_{w}$. These values are assumed to be greater than the ambient temperature $T_{\infty}$ and the nanoparticle concentration $\phi_{\infty}$ at any arbitrary reference point in the medium.

The porous medium is considered to be homogeneous and isotropic and is saturated with a fluid which is in local thermodynamic equilibrium with the solid matrix. We assume the fluid properties to be constant except the density in the buoyancy term of the balance of momentum equation. The fluid is considered to be a gray, absorbing emitting radiation but nonscattering medium and the Rosseland approximation [28] is used to describe the radiative heat flux in the energy equation. 
The governing equations for this problem under the laminar boundary layer flow assumptions, Boussinesq approximation, and using the non-Darcy flow through a homogeneous porous medium near the inclined wavy surface are given by $[12,29,30]$

$$
\begin{aligned}
& \frac{\partial u}{\partial x}+\frac{\partial v}{\partial y}=0, \\
& \left(1+\frac{\widetilde{K}}{v} \sqrt{u^{2}+v^{2}}\right)\left[\frac{\partial u}{\partial y}-\frac{\partial v}{\partial x}\right]+\frac{\widetilde{K}}{v \sqrt{u^{2}+v^{2}}}\left[u^{2} \frac{\partial u}{\partial y}\right. \\
& \left.+u v\left(\frac{\partial v}{\partial y}-\frac{\partial u}{\partial x}\right)-v^{2} \frac{\partial v}{\partial x}\right] \\
& \quad=\frac{\left(1-\phi_{\infty}\right) \rho_{f_{\infty}} \beta K g}{\mu}\left(\frac{\partial T}{\partial y} \sin A-\frac{\partial T}{\partial x} \cos A\right) \\
& \quad-\frac{\left.\partial \rho_{p}-\rho_{f_{\infty}}\right) K g}{\mu}\left(\frac{\partial \phi}{\partial y} \sin A-\frac{\partial \phi}{\partial x} \cos A\right) \\
& +v \frac{\partial T}{\partial x}=\alpha\left(\frac{\partial^{2} T}{\partial x^{2}}+\frac{\partial^{2} T}{\partial y^{2}}\right) \\
& \left.+\frac{\partial^{2} T}{\partial y^{2}}\right), \\
& +\gamma\left[\frac{\partial \phi}{\partial y_{B}^{2}}=D_{B}\left(\frac{\partial \phi}{\partial x} \frac{\partial T}{\partial x}+\frac{\partial \phi}{\partial y} \frac{\partial T}{\partial y}\right)\right. \\
& \left.+\frac{D_{T}}{T_{\infty}}\left(\left(\frac{\partial T}{\partial x}\right)^{2}+\left(\frac{\partial T}{\partial y}\right)^{2}\right)\right]+\frac{16 \sigma T_{\infty}^{3}}{3 K_{e}}\left(\frac{\partial^{2} T}{\partial x^{2}}\right.
\end{aligned}
$$

where $u$ and $v$ are the velocity components in the $x$ and $y$ directions, respectively, $T$ is the temperature, $\phi$ is the nanoparticle concentration, $g$ is the acceleration due to gravity, $K$ is the permeability, $\rho_{f}$ is the the density of the base fluid, $\rho_{p}$ is the density of the particles, $\rho C_{p}$ is the heat capacitance of the nanoparticles, $v$ is the kinematic viscosity of the fluid, $\alpha=k /(\rho c)_{f}$ is the effective thermal diffusivity, $\beta$ is the volumetric thermal expansion coefficient of the nanofluid, $\mu$ is the dynamic viscosity of the fluid, $D_{B}$ is the Brownian diffusion coefficient, $D_{T}$ is the thermophoretic diffusion coefficient, $\gamma$ is the ratio between the effective heat capacity of the nanoparticle material and heat capacity of the fluid (i.e., $\left.\gamma=\varepsilon(\rho c)_{p} /(\rho c)_{f}\right), K_{e}$ and $\sigma$ are the mean absorption coefficient and Stefan-Boltzmann constant, and $\widetilde{K}$ is a material parameter which is a measure of inertia impedance of the matrix to account for non-Darcian inertial effects.
The boundary conditions are

$$
\begin{aligned}
& v=0, \\
& T=T_{w}, \\
& \phi=\phi_{w}, \\
& u=U_{\infty}, \\
& T \longrightarrow T_{\infty}, \\
& \phi \longrightarrow \phi_{\infty}
\end{aligned}
$$

$$
\text { at } y=\delta(x) \text {, }
$$

$$
\text { as } y \longrightarrow \infty \text {. }
$$

Introducing the stream function $\psi$ by

$$
\begin{aligned}
& u=\frac{\partial \psi}{\partial y} \\
& v=-\frac{\partial \psi}{\partial x}
\end{aligned}
$$

and the following nondimensional variables

$$
\begin{aligned}
(\widehat{x}, \widehat{y}, \widehat{a}, \widehat{\delta}) & =l^{-1}(x, y, a, \delta), \\
\theta & =\frac{T-T_{\infty}}{T_{w}-T_{\infty}}, \\
\widehat{\psi} & =\frac{\psi}{U_{\infty} l}, \\
s & =\frac{\phi-\phi_{\infty}}{\phi_{w}-\phi_{\infty}}
\end{aligned}
$$

in (3)-(5), we get the following system of nondimensional equations:

$$
\begin{aligned}
& \left(1+F_{c} Q\right)\left(\frac{\partial^{2} \widehat{\psi}}{\partial \widehat{x}^{2}}+\frac{\partial^{2} \widehat{\psi}}{\partial \widehat{y}^{2}}\right)+\frac{F_{c}}{Q}\left[\left(\frac{\partial \widehat{\psi}}{\partial \widehat{y}}\right)^{2} \frac{\partial^{2} \widehat{\psi}}{\partial \widehat{y}^{2}}\right. \\
& \left.+2 \frac{\partial \widehat{\psi}}{\partial \widehat{x}} \frac{\partial \widehat{\psi}}{\partial \widehat{y}} \frac{\partial^{2} \widehat{\psi}}{\partial \widehat{x} \partial \widehat{y}}+\left(\frac{\partial \widehat{\psi}}{\partial \widehat{x}}\right)^{2} \frac{\partial^{2} \widehat{\psi}}{\partial \widehat{x}^{2}}\right] \\
& =\Delta\left[\left(\frac{\partial \theta}{\partial \widehat{y}} \sin A-\frac{\partial \theta}{\partial \widehat{x}} \cos A\right)\right. \\
& \left.\quad-N_{r}\left(\frac{\partial s}{\partial \widehat{y}} \sin A-\frac{\partial s}{\partial \widehat{x}} \cos A\right)\right], \\
& \frac{\partial \widehat{\psi}}{\partial \widehat{y}} \frac{\partial \theta}{\partial \widehat{x}}-\frac{\partial \widehat{\psi}}{\partial \widehat{x}} \frac{\partial \theta}{\partial \widehat{y}}=\frac{1}{\mathrm{Pe}}\left[\left(1+\frac{4 R}{3}\right)\left(\frac{\partial^{2} \theta}{\partial \widehat{x}^{2}}+\frac{\partial^{2} \theta}{\partial \widehat{y}^{2}}\right)\right. \\
& \quad+N_{b}\left(\frac{\partial s}{\partial \widehat{x}} \frac{\partial \theta}{\partial \widehat{x}}+\frac{\partial s}{\partial \widehat{y}} \frac{\partial \theta}{\partial \widehat{y}}\right) \\
& \left.\quad+N_{t}\left(\left(\frac{\partial \theta}{\partial \widehat{x}}\right)^{2}+\left(\frac{\partial \theta}{\partial \widehat{y}}\right)^{2}\right)\right],
\end{aligned}
$$




$$
\begin{aligned}
& \frac{\partial \widehat{\psi}}{\partial \hat{y}} \frac{\partial s}{\partial \widehat{x}}-\frac{\partial \widehat{\psi}}{\partial \hat{x}} \frac{\partial s}{\partial \widehat{y}}=\frac{1}{\operatorname{Le} \operatorname{Pe}}\left[\left(\frac{\partial^{2} s}{\partial \widehat{x}^{2}}+\frac{\partial^{2} s}{\partial \hat{y}^{2}}\right)\right. \\
& \left.+\frac{N_{t}}{N_{b}}\left(\frac{\partial^{2} \theta}{\partial \hat{x}^{2}}+\frac{\partial^{2} \theta}{\partial \hat{y}^{2}}\right)\right],
\end{aligned}
$$

where $Q=\left[(\partial \widehat{\psi} / \partial \widehat{x})^{2}+(\partial \widehat{\psi} / \partial \widehat{y})^{2}\right]^{1 / 2}$ is the nondimensional velocity, $\mathrm{Ra}=\left(1-\phi_{\infty}\right) \rho_{f_{\infty}} \beta K g\left(T_{w}-T_{\infty}\right) l / \mu \alpha$ is the Rayleigh number, $\mathrm{Pe}=U_{\infty} l / \alpha$ is the Peclet number, $\Delta=\mathrm{Ra} / \mathrm{Pe}$ is the mixed convection number, $F_{c}=\widetilde{K} U_{\infty} / \nu$ is the non-Darcy parameter, $N_{r}=\left(\rho_{p}-\rho_{f_{\infty}}\right)\left(\phi_{w}-\phi_{\infty}\right) / \rho_{f_{\infty}} \beta\left(T_{w}-T_{\infty}\right)(1-$ $\left.\phi_{\infty}\right)$ is the buoyancy ratio, $R=4 \sigma T_{\infty}^{3} / K K_{e}$ is the Radiation parameter, $N_{b}=\gamma D_{B}\left(\phi_{w}-\phi_{\infty}\right) / \alpha$ is the Brownian motion parameter, $N_{t}=\gamma D_{T}\left(T_{w}-T_{\infty}\right) / \alpha T_{\infty}$ is the thermophoresis parameter, and $\mathrm{Le}=\alpha / D_{B}$ is the Lewis number.

The effect of the wavy surface can be transferred from the boundary conditions into the governing equations by the coordinate transformation

$$
\begin{aligned}
\xi & =\widehat{x}, \\
\eta & =\frac{(\widehat{y}-\delta) \mathrm{Pe}^{1 / 2}}{\xi^{1 / 2}\left(1+\dot{\delta}^{2}\right)}, \\
\widehat{\psi} & =\mathrm{Pe}^{1 / 2} \xi^{1 / 2} f(\xi, \eta) .
\end{aligned}
$$

Substituting (10) into (9) and letting $\mathrm{Ra} \rightarrow \infty$ (i.e., boundary layer approximation), we obtain the following equations:

$$
\begin{aligned}
f^{\prime \prime} & +2 F_{c}\left(1+\dot{\delta}^{2}\right)^{-1 / 2} f^{\prime} f^{\prime \prime} \\
& =\Delta(\sin A+\dot{\delta} \cos A)\left(\theta^{\prime}-N_{r} s^{\prime}\right), \\
& \left(1+\frac{4 R}{3}\right) \theta^{\prime \prime}+\frac{1}{2} f \theta^{\prime}+N_{b} s^{\prime} \theta^{\prime}+N_{t} \theta^{\prime 2}=0, \\
s^{\prime \prime} & +\frac{1}{2} \operatorname{Le} f s^{\prime}+\frac{N_{t}}{N_{b}} \theta^{\prime \prime}=0 .
\end{aligned}
$$

The associated boundary conditions are

$$
\begin{aligned}
& f=0, \\
& \theta=1, \\
& s=1,
\end{aligned}
$$

at $\eta=0$,

$$
\begin{gathered}
f^{\prime} \longrightarrow 1 \\
\theta \longrightarrow 0 \\
s \longrightarrow 0
\end{gathered}
$$

as $\eta \longrightarrow \infty$.

The primary objective of this study is to estimate the parameters of engineering interest in fluid flow, heat, and nanoparticle mass transport problems, namely, the Nusselt number $\mathrm{Nu}_{x}$ and nanoparticle Sherwood number $\mathrm{NSh}_{x}$. These parameters characterize the wall heat and nanoparticle mass transfer rates, respectively.

The local heat and nanoparticle mass fluxes from the wavy plate can be obtained from

$$
\begin{aligned}
& q_{w}=-k n \cdot \nabla T+n \cdot q_{r}, \\
& q_{n p}=-D_{B} n \cdot \nabla \phi,
\end{aligned}
$$

where $n=\left(-\dot{\delta} / \sqrt{1+\dot{\delta}^{2}}, 1 / \sqrt{1+\dot{\delta}^{2}}\right)$ is the unit normal vector to the wavy plate and $q_{r}$ is the radiative heat flux given by $q_{r}=\left(-4 \sigma / 3 K_{e}\right) \nabla T^{4}$.

The dimensionless local Nusselt number $\mathrm{Nu}_{x}=$ $x q_{w} / k\left(T_{w}-T_{\infty}\right)$ and the nanoparticle Sherwood number $\mathrm{NSh}_{x}=x q_{n p} / D_{B}\left(\phi_{w}-\phi_{\infty}\right)$ are given by

$$
\begin{aligned}
& \frac{\mathrm{Nu}_{x}}{\sqrt{\mathrm{Pe}_{x}}}=-\left(1+\frac{4 R}{3}\right) \sqrt{\frac{1}{1+\dot{\delta}^{2}}}\left(\frac{\partial \theta}{\partial \eta}\right)_{\eta=0}, \\
& \frac{\mathrm{NSh}_{x}}{\sqrt{\mathrm{Pe}_{x}}}=-\sqrt{\frac{1}{1+\dot{\delta}^{2}}}\left(\frac{\partial s}{\partial \eta}\right)_{\eta=0} .
\end{aligned}
$$

\section{Numerical Solution}

Equations (11) along with the boundary conditions (12a) and (12b) were solved numerically using the successive linearization method (SLM) [31-33]. Using this method the nonlinear boundary layer equations reduce to a system of linear differential equations. The Chebyshev pseudo spectral method is then used to transform the iterative sequence of linearized differential equations into a system of linear algebraic equations which are converted into a matrix system. In this method we assume that the independent variables $f(\eta), \theta(\eta)$, and $s(\eta)$ can be expressed as

$$
\begin{aligned}
& f(\eta)=f_{i}(\eta)+\sum_{n=0}^{i-1} f_{n}(\eta), \\
& \theta(\eta)=\theta_{i}(\eta)+\sum_{n=0}^{i-1} \theta_{n}(\eta), \\
& s(\eta)=s_{i}(\eta)+\sum_{n=0}^{i-1} s_{n}(\eta),
\end{aligned}
$$

where $f_{i}, \theta_{i}$, and $s_{i},(i=1,2,3, \ldots)$ are unknown functions and $f_{n}, \theta_{n}$, and $s_{n}$ are the approximations which are obtained by recursively solving the linear part of the equation system that results from substituting (15) in (11) and neglecting the nonlinear terms in $f_{i}, \theta_{i}$, and $s_{i}$

$$
\begin{array}{r}
a_{1, i-1} f_{i}^{\prime \prime}+a_{2, i-1} f_{i}^{\prime}+a_{3, i-1} \theta_{i}^{\prime}+a_{4, i-1} s_{i}^{\prime}=r_{1, i-1}, \\
b_{1, i-1} \theta_{i}^{\prime \prime}+b_{2, i-1} \theta_{i}^{\prime}+b_{3, i-1} s_{i}^{\prime}+b_{4, i-1} f_{i}=r_{2, i-1}, \\
s_{i}^{\prime \prime}+c_{1, i-1} s_{i}^{\prime}+c_{2, i-1} \theta_{i}^{\prime \prime}+c_{3, i-1} f_{i}=r_{3, i-1},
\end{array}
$$


where

$$
\begin{aligned}
a_{1, i-1}= & 1+2 F_{c}\left(1+\dot{\delta}^{2}\right)^{-1 / 2} \sum_{n=0}^{i-1} f_{n}^{\prime}, \\
a_{2, i-1}= & 2 F_{c}\left(1+\dot{\delta}^{2}\right)^{-1 / 2} \sum_{n=0}^{i-1} f_{n}^{\prime \prime}, \\
a_{3, i-1}= & -\Delta(\sin A+\dot{\delta} \cos A), \\
a_{4, i-1}= & N_{r} \Delta(\sin A+\dot{\delta} \cos A), \\
b_{1, i-1}= & 1+\frac{4 R}{3}, \\
b_{2, i-1}= & \frac{1}{2} \sum_{n=0}^{i-1} f_{n}+N_{b} \sum_{n=0}^{i-1} s_{n}^{\prime}+2 N_{t} \sum_{n=0}^{i-1} \theta_{n}^{\prime}, \\
b_{3, i-1}= & N_{b} \sum_{n=0}^{i-1} \theta_{n}^{\prime}, \\
& -N_{b} \sum_{n=0}^{i-1} s_{n}^{\prime} \sum_{n=0}^{i-1} \theta_{n}^{\prime}-N_{t} \sum_{n=0}^{i-1} \theta_{n}^{\prime} \sum_{n=0}^{i-1} \theta_{n}^{\prime}, \\
r_{3, i-1}= & -\sum_{n=0}^{i-1} s_{n}^{\prime \prime}-\frac{1}{2} \sum_{n=0}^{i-1} f_{n} \sum_{n=0}^{i-1} s_{n}^{\prime}-\frac{N_{t}}{N_{b}} \sum_{n=0}^{i-1} \theta_{n}^{\prime \prime} . \\
& +\Delta(\sin A+\dot{\delta} \cos A) \sum_{n=0}^{i-1} \theta_{n}^{\prime} \\
r_{2, i-1}= & -\left(1+\frac{4 R}{3}\right) \sum_{n=0}^{i-1} \theta_{n}^{\prime \prime}-\frac{1}{2} \sum_{n=0}^{i-1} f_{n} \sum_{n=0}^{i-1} \theta_{n}^{\prime} \\
c_{1, i-1}= & -\sum_{n=0}^{i-1} f_{n}, \\
\mathcal{C}_{3, i-1}= & \frac{1}{2} \operatorname{Lin} \sum_{n=0}^{i-1} s_{n}^{\prime}, \\
\mathcal{C}_{2, i-1}= & N_{t}, \\
N_{b} &
\end{aligned}
$$

The boundary conditions reduce to

$$
\begin{aligned}
& f_{i}(0)=f_{i}^{\prime}(\infty)=0, \\
& \theta_{i}(0)=\theta_{i}(\infty)=s_{i}(0)=s_{i}(\infty)=0 .
\end{aligned}
$$

The initial approximations $f_{0}(\eta), \theta_{0}(\eta)$, and $s_{0}(\eta)$ are chosen such that they satisfy the boundary conditions (12a) and (12b) and are taken as $f_{0}(\eta)=1+\eta-e^{-\eta}, \theta_{0}(\eta)=e^{-\eta}$, and $s_{0}(\eta)=e^{-\eta}$.

The solutions $f_{i}, \theta_{i}$, and $s_{i}(i \geq 1)$ are obtained by iteratively solving (16). The approximate solutions for $f(\eta)$, $\theta(\eta)$, and $s(\eta)$ are then obtained as

$$
\begin{aligned}
& f(\eta) \approx \sum_{m=0}^{M} f_{m}(\eta), \\
& \theta(\eta) \approx \sum_{m=0}^{M} \theta_{m}(\eta), \\
& s(\eta) \approx \sum_{m=0}^{M} s_{m}(\eta),
\end{aligned}
$$

where $M$ is the order of SLM approximation. The linearized equations (16) are solved using the Chebyshev spectral collocation method [34]. In this method the unknown functions are approximated by the Chebyshev interpolating polynomials in such a way that they are collocated at the Gauss-Lobatto points defined as

$$
\xi_{j}=\cos \frac{\pi j}{N}, \quad j=0,1,2, \ldots, N,
$$

where $N$ is the number of collocation points used. The physical region $[0, \infty)$ is transformed into the region $[-1,1]$ using the domain truncation technique in which the problem is solved on the interval $[0, L]$ instead of $[0, \infty)$. This leads to the mapping

$$
\frac{\eta}{L}=\frac{\xi+1}{2}, \quad-1 \leq \xi \leq 1,
$$

where $L$ is a scaling parameter used to invoke the boundary condition at infinity. The functions $f_{i}, \theta_{i}$, and $S_{i}$ are approximated at the collocation points by

$$
\begin{aligned}
& f_{i}(\xi)=\sum_{k=0}^{N} f_{i}\left(\xi_{k}\right) T_{k}\left(\xi_{j}\right), \\
& \theta_{i}(\xi)=\sum_{k=0}^{N} \theta_{i}\left(\xi_{k}\right) T_{k}\left(\xi_{j}\right), \\
& s_{i}(\xi)=\sum_{k=0}^{N} s_{i}\left(\xi_{k}\right) T_{k}\left(\xi_{j}\right),
\end{aligned}
$$

$$
j=0,1,2, \ldots, N,
$$

where $T_{k}$ is the $k$ th Chebyshev polynomial defined by

$$
T_{k}(\xi)=\cos \left[k \cos ^{-1} \xi\right] \text {. }
$$


The derivatives of the variables at the collocation points are represented as

$$
\begin{aligned}
& \frac{d^{a} f_{i}}{d \eta^{a}}=\sum_{k=0}^{N} \mathbf{D}_{k j}^{a} f_{i}\left(\xi_{k}\right), \\
& \frac{d^{a} \theta_{i}}{d \eta^{a}}=\sum_{k=0}^{N} \mathbf{D}_{k j}^{a} \theta_{i}\left(\xi_{k}\right), \\
& \frac{d^{a} s_{i}}{d \eta^{a}}=\sum_{k=0}^{N} \mathbf{D}_{k j}^{a} s_{i}\left(\xi_{k}\right),
\end{aligned}
$$

$$
j=0,1,2, \ldots, N
$$

where $a$ is the order of differentiation and $\mathbf{D}=(2 / L) \mathscr{D}$ with $\mathscr{D}$ being the Chebyshev spectral differentiation matrix. Substituting (21)-(24) into (16) leads to the matrix equation

$$
\mathbf{A}_{i-1} \mathbf{X}_{i}=\mathbf{R}_{i-1},
$$

subject to the boundary conditions

$$
\begin{aligned}
f_{i}\left(\xi_{N}\right) & =0, \\
\sum_{k=0}^{N} \mathbf{D}_{0 k} f_{i}\left(\xi_{k}\right) & =0 \\
\theta_{i}\left(\xi_{N}\right) & =\theta_{i}\left(\xi_{0}\right)=s_{i}\left(\xi_{N}\right)=s_{i}\left(\xi_{0}\right)=0 .
\end{aligned}
$$

In (25), $\mathbf{A}_{i-1}$ is a $(3 N+3) \times(3 N+3)$ square matrix and $\mathbf{X}_{i}$ and $\mathbf{R}_{i-1}$ are $(3 N+3) \times 1$ column vectors defined by

$$
\begin{aligned}
\mathbf{A}_{i-1} & =\left[\begin{array}{lll}
A_{11} & A_{12} & A_{13} \\
A_{21} & A_{22} & A_{23} \\
A_{31} & A_{32} & A_{33}
\end{array}\right], \\
\mathbf{X}_{i} & =\left[\begin{array}{l}
\mathbf{F}_{i} \\
\boldsymbol{\Theta}_{i} \\
\boldsymbol{\Phi}_{i}
\end{array}\right], \\
\mathbf{R}_{i-1} & =\left[\begin{array}{l}
\mathbf{r}_{1, i-1} \\
\mathbf{r}_{2, i-1} \\
\mathbf{r}_{3, i-1}
\end{array}\right],
\end{aligned}
$$

where

$$
\begin{aligned}
& \mathbf{F}_{i}=\left[f_{i}\left(\xi_{0}\right), f_{i}\left(\xi_{1}\right), \ldots, f_{i}\left(\xi_{N-1}\right), f_{i}\left(\xi_{N}\right)\right]^{T}, \\
& \Theta_{i}=\left[\theta_{i}\left(\xi_{0}\right), \theta_{i}\left(\xi_{1}\right), \ldots, \theta_{i}\left(\xi_{N-1}\right), \theta_{i}\left(\xi_{N}\right)\right]^{T}, \\
& \Phi_{i}=\left[s_{i}\left(\xi_{0}\right), s_{i}\left(\xi_{1}\right), \ldots, s_{i}\left(\xi_{N-1}\right), s_{i}\left(\xi_{N}\right)\right]^{T},
\end{aligned}
$$

TABLE 1: Comparison of values of $-\theta^{\prime}(0)$ for aiding and opposing flow by the present method and Ping [35] for fixed values of $A=\pi / 2$, $a=0, \xi=0, N_{r}=0, N_{t}=0, N_{b} \rightarrow 0$, Le $=0$, and $R=0$.

\begin{tabular}{lccccc}
\hline & \multicolumn{2}{c}{ Aiding flow } & \multicolumn{3}{c}{ Opposing flow } \\
$\Delta$ & Ping [35] & Present & $\Delta$ & Ping [35] & Present \\
\hline 0 & 0.5641 & 0.56415775 & -0.2 & 0.5269 & 0.52691089 \\
0.5 & 0.6473 & 0.64736510 & -0.4 & 0.4865 & 0.48653284 \\
1 & 0.7205 & 0.72055401 & -0.6 & 0.442 & 0.44202064 \\
3 & 0.9574 & 0.95744512 & -0.8 & 0.3916 & 0.39166292 \\
10 & 1.516 & 1.51623967 & -1.0 & 0.332 & 0.33202116 \\
20 & 2.066 & 2.066 & & & \\
\hline
\end{tabular}

$$
\begin{aligned}
& \mathbf{r}_{1, i-1}=\left[r_{1, i-1}\left(\xi_{0}\right), r_{1, i-1}\left(\xi_{1}\right), \ldots, r_{1, i-1}\left(\xi_{N-1}\right),\right. \\
&\left.r_{1, i-1}\left(\xi_{N}\right)\right]^{T}, \mathbf{r}_{2, i-1}=\left[r_{2, i-1}\left(\xi_{0}\right), r_{2, i-1}\left(\xi_{1}\right), \ldots, r_{2, i-1}\left(\xi_{N-1}\right),\right. \\
&\left.r_{2, i-1}\left(\xi_{N}\right)\right]^{T}, \\
& \mathbf{r}_{3, i-1}=\left[r_{3, i-1}\left(\xi_{0}\right), r_{3, i-1}\left(\xi_{1}\right), \ldots, r_{3, i-1}\left(\xi_{N-1}\right),\right. \\
&\left.r_{3, i-1}\left(\xi_{N}\right)\right]^{T}, \\
& A_{11}=a_{1, i-1} \mathbf{D}^{2}+a_{2, i-1} \mathbf{D}, \\
& A_{12}=a_{3, i-1} \mathbf{D}, \\
& A_{13}=a_{4, i-1} \mathbf{D}, \\
& A_{21}=b_{4, i-1} \mathbf{I}, \\
& A_{22}=b_{1, i-1} \mathbf{D}^{2}+b_{2, i-1} \mathbf{D}, \\
& A_{23}=b_{3, i-1} \mathbf{D}, \\
& A_{31}=c_{3, i-1} \mathbf{I}, \\
& A_{32}=c_{2, i-1} \mathbf{D}^{2}, \\
& A_{33}=\mathbf{D}^{2}+c_{1, i-1} \mathbf{D} .
\end{aligned}
$$

Here $a_{k, i-1}, b_{l, i-1}$, and $c_{l, i-1}((k=1,2)$ and $(l=1,2,3))$ are diagonal matrices of size $(N+1) \times(N+1)$ and $\mathbf{I}$ is an identity matrix of size $(N+1) \times(N+1)$. After modifying the matrix system (25) to incorporate boundary conditions (26), the solution is obtained as

$$
\mathbf{X}_{i}=\mathbf{A}_{i-1}^{-1} \mathbf{R}_{i-1} .
$$

\section{Results and Discussion}

In order to validate the numerical procedure generated, the results of the present problem have been compared with work of Ping [35] as a special case by taking $A=\pi / 2, a=0, \xi=0$, $N_{r}=0, N_{t}=0, N_{b} \rightarrow 0$, Le $=0, F_{c}=0$, and $R=0$ and we found that they are in good agreement, as shown in Table 1. Numerical solutions for the dimensionless velocity, temperature and nanoparticle volume fraction functions, and 


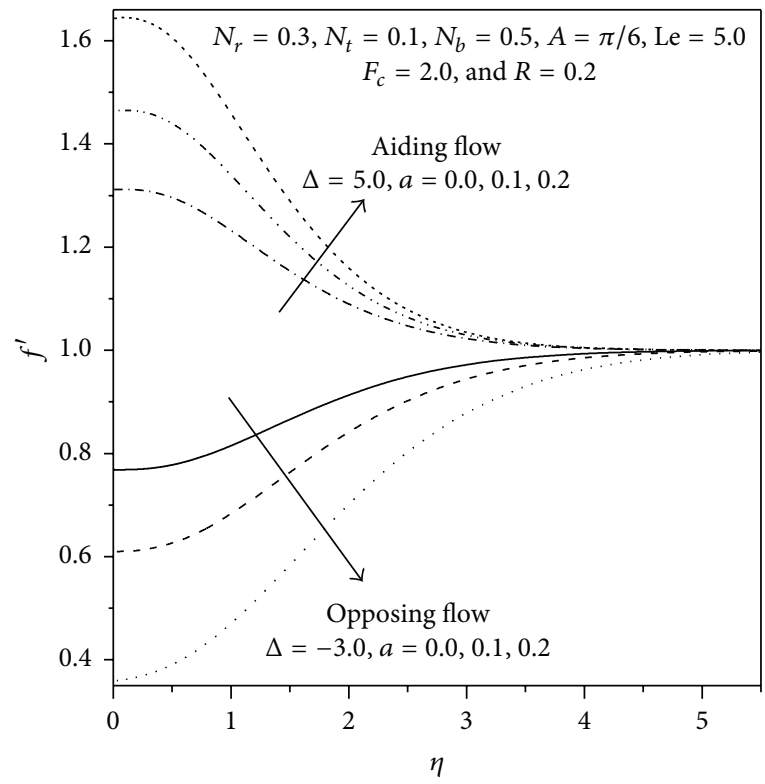

(a)

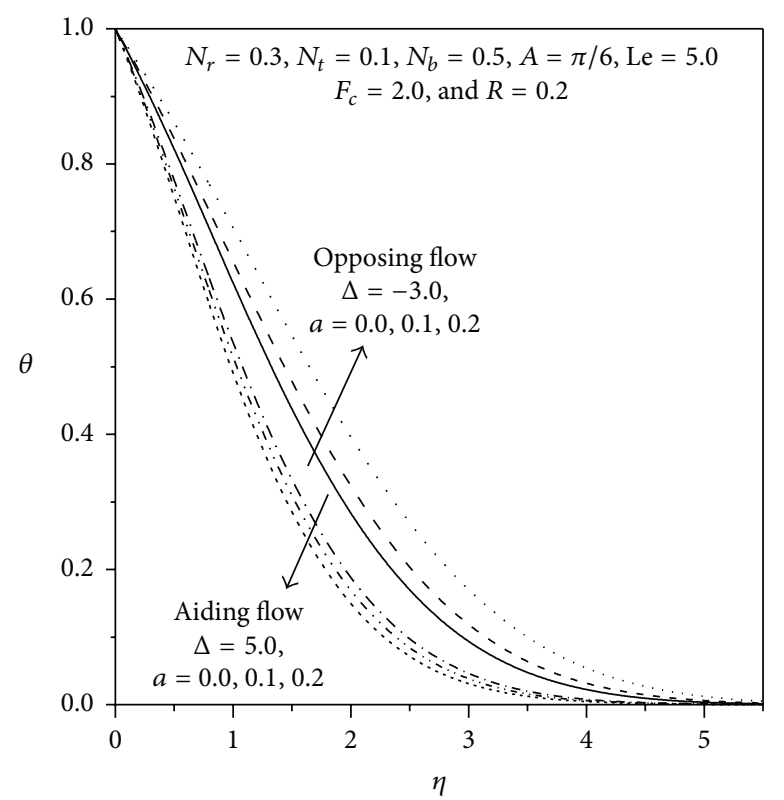

(b)

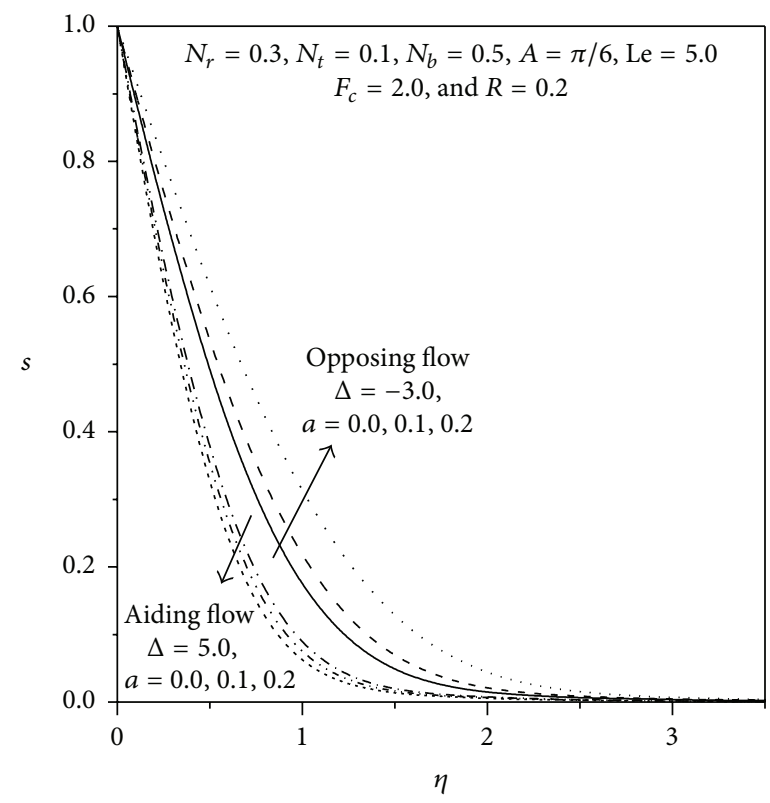

(c)

Figure 2: Variation of (a) velocity, (b) temperature, and (c) nanoparticle volume fraction profiles with the wave amplitude (a).

heat and nanoparticle mass transfer rates are computed and presented graphically in Figures 2-11. The effects of radiation $R$, angle of inclination $A$, Brownian motion parameter $N_{b}$, thermophoretic parameter $N_{t}$, non-Darcy parameter $F_{c}$, and amplitude $a$ of the wavy surface have been discussed.

Figure 2 shows the effect of the amplitude of the wavy surface on velocity, temperature, and nanoparticle volume fraction distributions. It is observed that as $a$ increases, velocity increases near the plate and decreases away from the plate, whereas the temperature and nanoparticle volume fraction decrease for aiding flow and the reverse trend is observed in the case of opposing flow.
The effect of the angle of inclination of the wavy surface on velocity, temperature, and nanoparticle volume fraction is plotted in Figure 3. The similarity equations for the limiting cases of the horizontal and vertical plates are recovered from the transformed equations by setting $A=0^{\circ}$ and $A=90^{\circ}$, respectively. It is noted from Figures 3(a)-3(c) that as $A$ increases, the velocity increases near the plate and decreases away from the plate for aiding flow whereas the velocity decreases near the plate and increases away from the plate for opposing flow. But the temperature and nanoparticle volume fraction decrease within the boundary layer region for the aiding flow and increase for opposing 


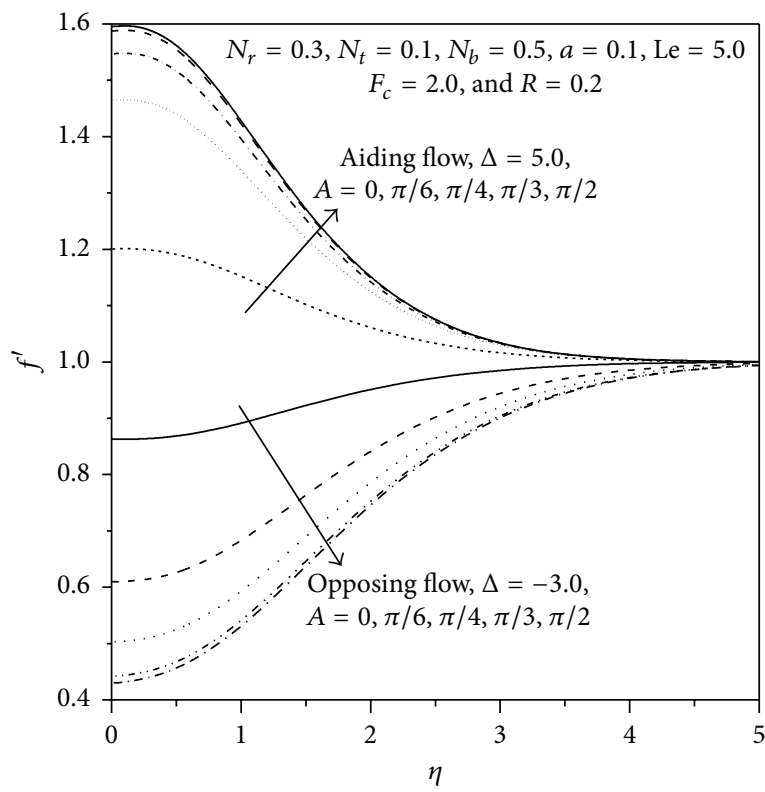

(a)

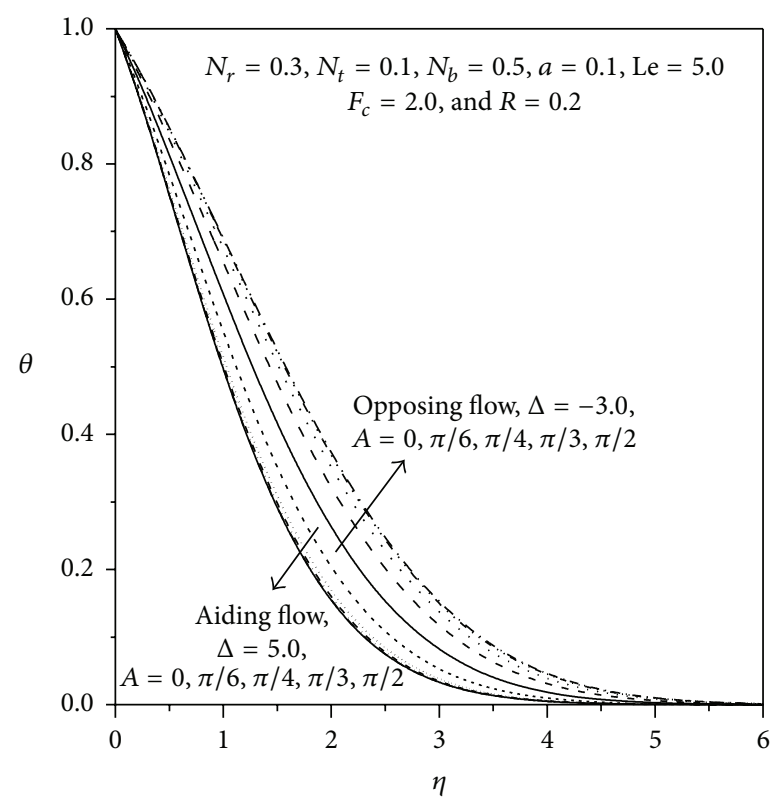

(b)

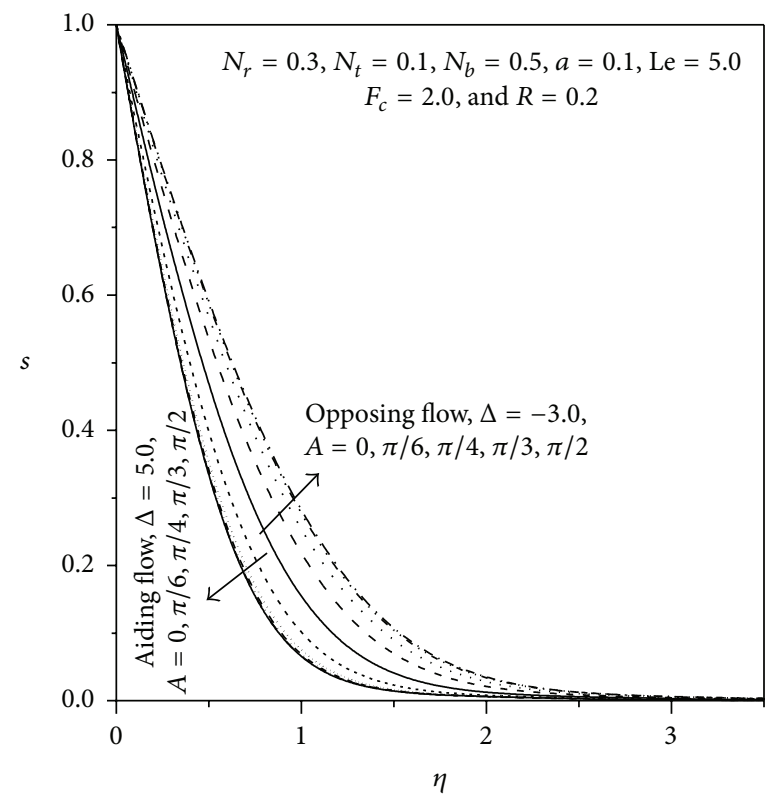

(c)

FIGURE 3: Variation of (a) velocity, (b) temperature, and (c) nanoparticle volume fraction profiles with angle of inclination $(A)$.

flow. When the surface is vertical, the smallest temperature and nanoparticle volume fraction distributions are observed for aiding flow whereas largest temperature and nanoparticle volume fraction distributions are observed for opposing flow. While for the horizontal surface, largest temperature and nanoparticle volume fraction distributions are observed for aiding flow whereas smallest temperature and nanoparticle volume fraction distributions are observed for opposing flow.

Figure 4 displays the effect of non-Darcy parameter on the dimensionless velocity, temperature, and nanoparticle fraction. Figure 4(a) shows that an increase in non-Darcy parameter reduces velocity for aiding flow. Since $F_{c}$ represent the inertial drag, thus an increase in the non-Darcy parameter increases the resistance to the flow and so a decrease in the fluid velocity is ensured. Here $F_{c}=0$ represents the case where the flow is Darcian. The velocity is maximum in this case due to the total absence of inertial drag. In case of opposing flow the velocity is increasing with the increase in the non-Darcy parameter. An increase in $F_{c}$ increases temperature values for aiding flow, since as the fluid is decelerated, energy is dissipated as heat and serves to increase temperatures. As such the temperature is minimised for the lowest value of $F_{c}$ and maximised for the highest value of $F_{c}$ as shown in Figure 4(b). Further it is noticed that 


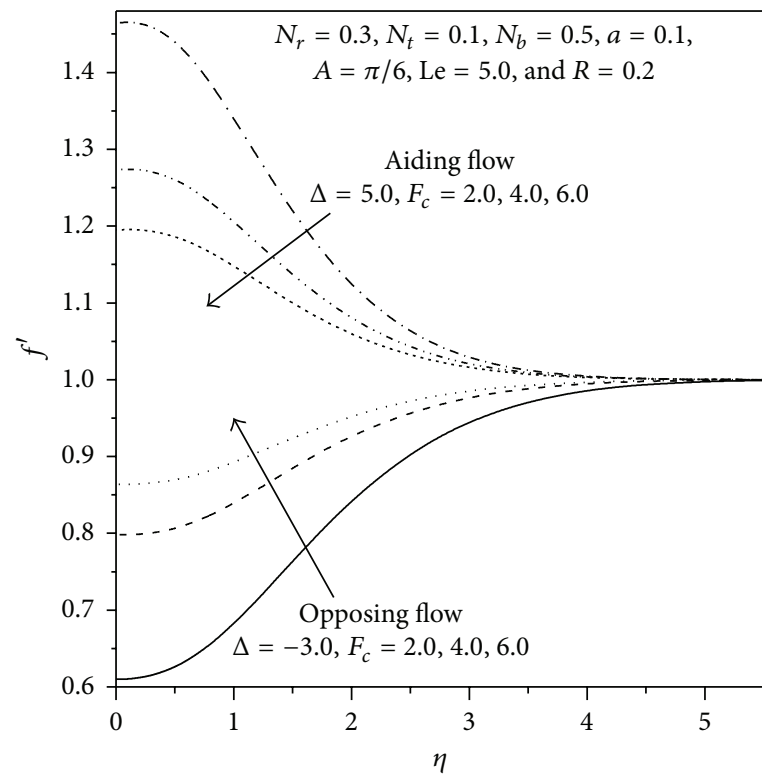

(a)

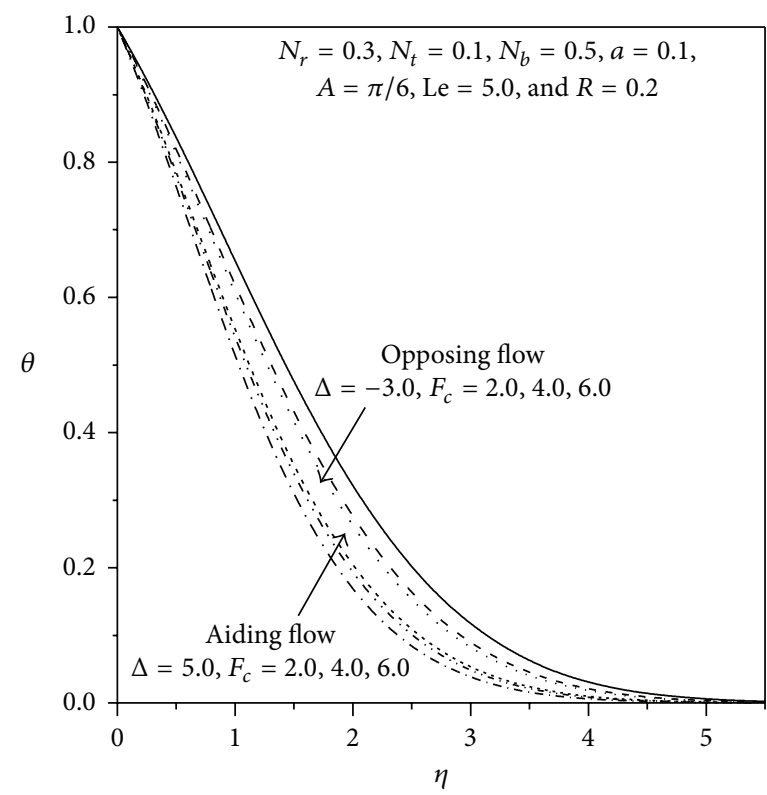

(b)

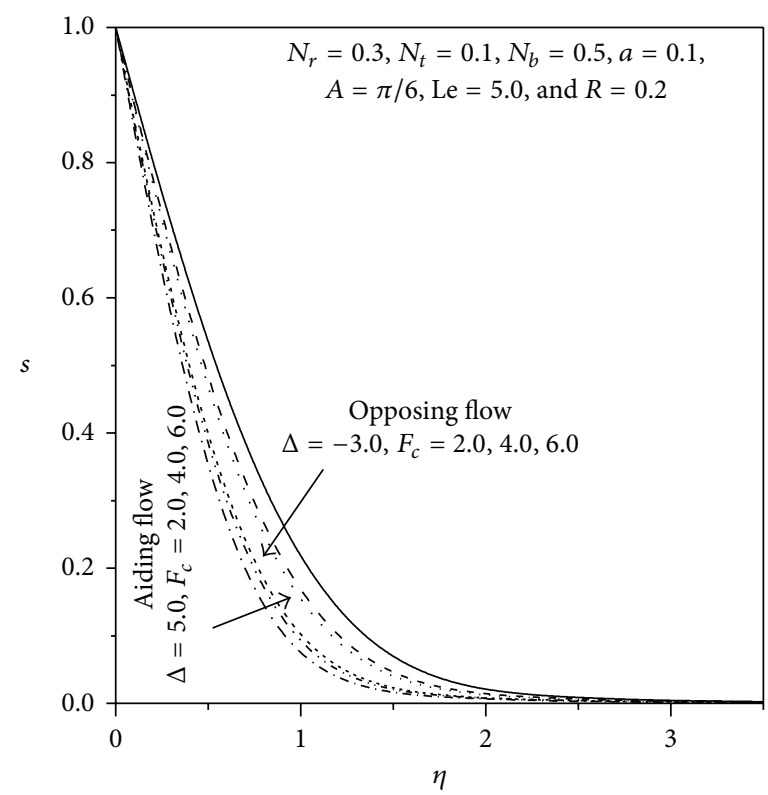

(c)

FIGURE 4: Variation of (a) velocity, (b) temperature, and (c) nanoparticle volume fraction profiles with non-Darcy parameter $\left(F_{c}\right)$.

increase in $F_{c}$ decreases the temperature for opposing flow. From Figure 4(c), it is observed that the nanoparticle volume fraction increases for aiding flow and decreases in the case of opposing flow.

The effect of radiation $R$ on velocity, temperature, and nanoparticle volume fraction is shown in Figure 5. Figure 5(a) reveals that an increase in the radiation parameter increases the velocity for aiding flow and reduces the same for the case of opposing flow. Increase in radiation parameter enhances the temperature for both aiding and opposing flows as shown in Figure 5(b). We observe a negligible effect of radiation on nanoparticle volume fraction as shown in
Figure 5(c). These results can be explained by the fact that an increase in the radiation parameter $\left(R=4 \sigma T_{\infty}^{3} / K K_{e}\right)$ for given $K$ and $T_{\infty}$ means a decrease in the Rosseland radiation absorptivity $K_{e}$. Hence, the divergence of radiative heat flux $q_{r}$ increases as $K_{e}$ decreases. Therefore, the rate of radiative heat transferred to the fluid increases, and consequently the fluid temperature and simultaneously the velocity of the fluid also increase.

Figure 6 shows the effect of wave amplitude on the Nusselt and nanoparticle Sherwood number. It reveals that an increase in the wave amplitude reduces the local heat and nanoparticle mass transfer for both aiding and opposing 


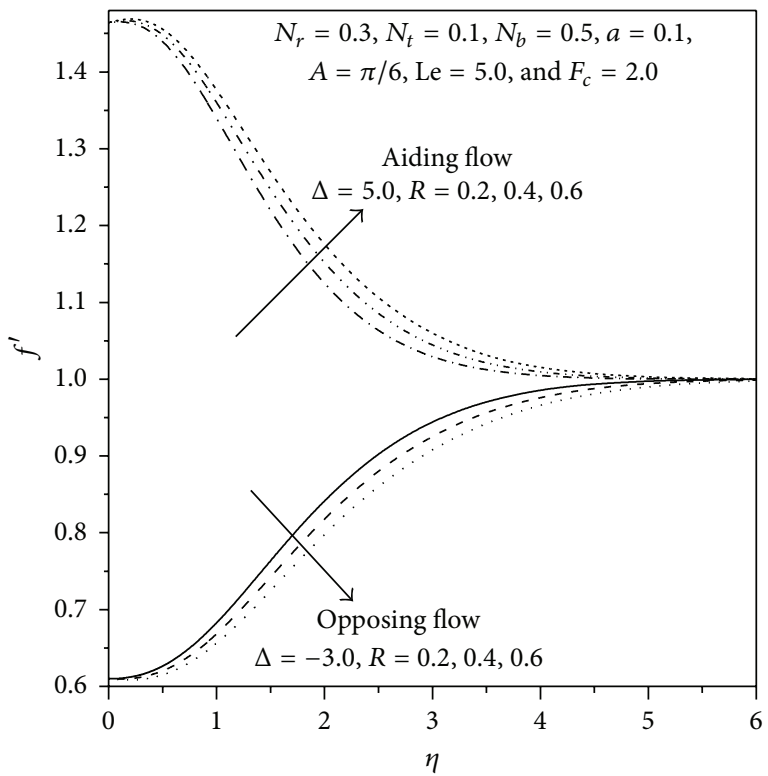

(a)

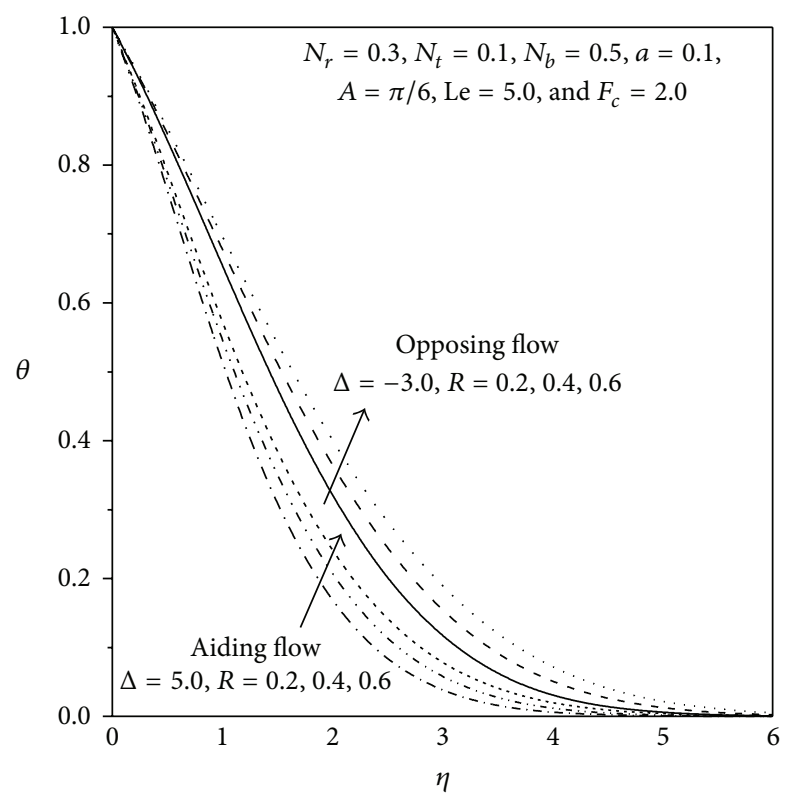

(b)

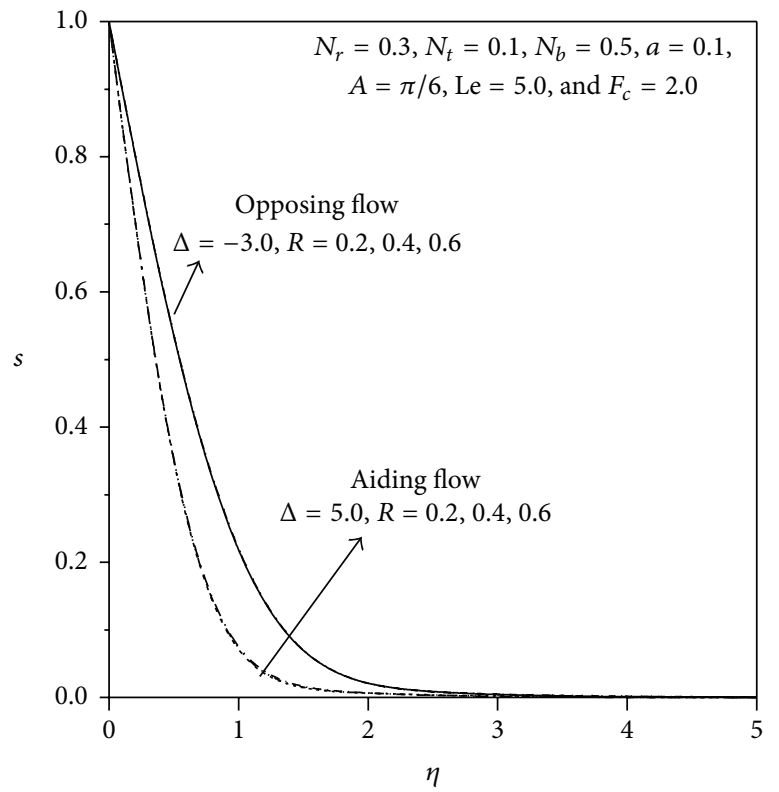

(c)

FIGURE 5: Variation of (a) velocity, (b) temperature, and (c) nanoparticle volume fraction profiles with radiation parameter $(R)$.

flows. In general, we conclude that increasing the wave amplitude makes the surface more roughened.

The variation of heat and nanoparticle mass transfer rates for various values of the angle of inclination $A$ is displayed in Figure 7. This figure shows that increasing the angle of inclination increases the buoyancy force and assists the flow, leading to an increase in the heat and nanoparticle mass transfer rates for aiding flow whereas reverse trend is observed in the case of opposing flow. The minimum values of the dimensionless heat and nanoparticle mass transfer rates are observed when the surface is horizontal; in which case, the buoyancy force is at its maximum. Therefore, the heat and nanoparticle mass transfer rates are at a lower level when this effect is considered $(A \neq 0)$.

The effect of Brownian motion parameter $N_{b}$ on the heat and nanoparticle mass transfer rates is presented in Figure 8. Figure 8(a) depicts that the dimensionless heat transfer rate decreases with the increase in the Brownian motion parameter for both aiding and opposing flows. An increase in the value of Brownian motion parameter enhances the nanoparticle volume fraction transfer rate for both aiding and opposing flows, as shown in Figure 8(b). Figure 9 depicts the streamwise distribution of Nusselt and Sherwood numbers for different values of thermophoretic parameter $N_{t}$. 


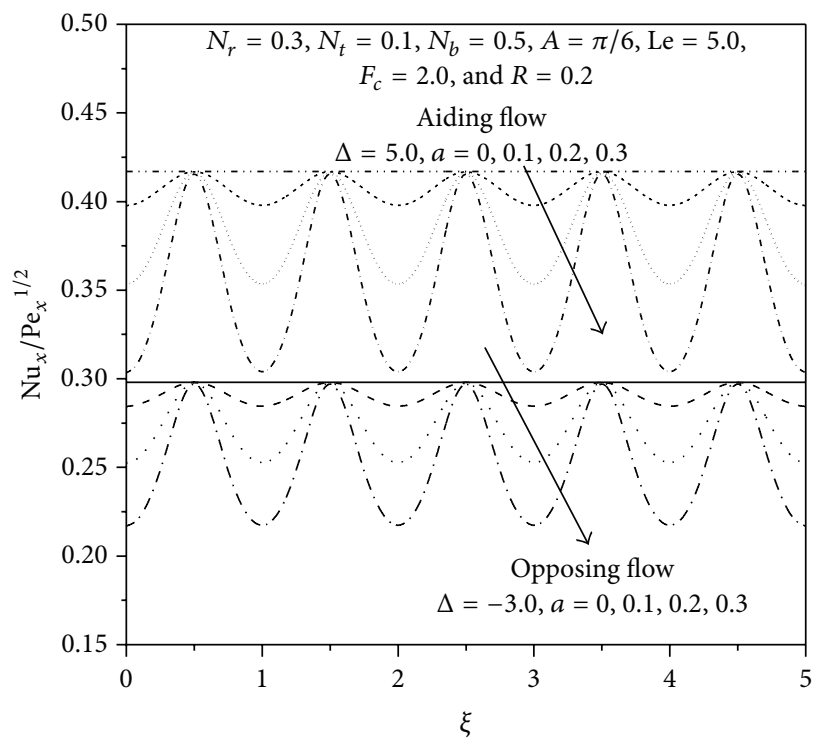

(a)

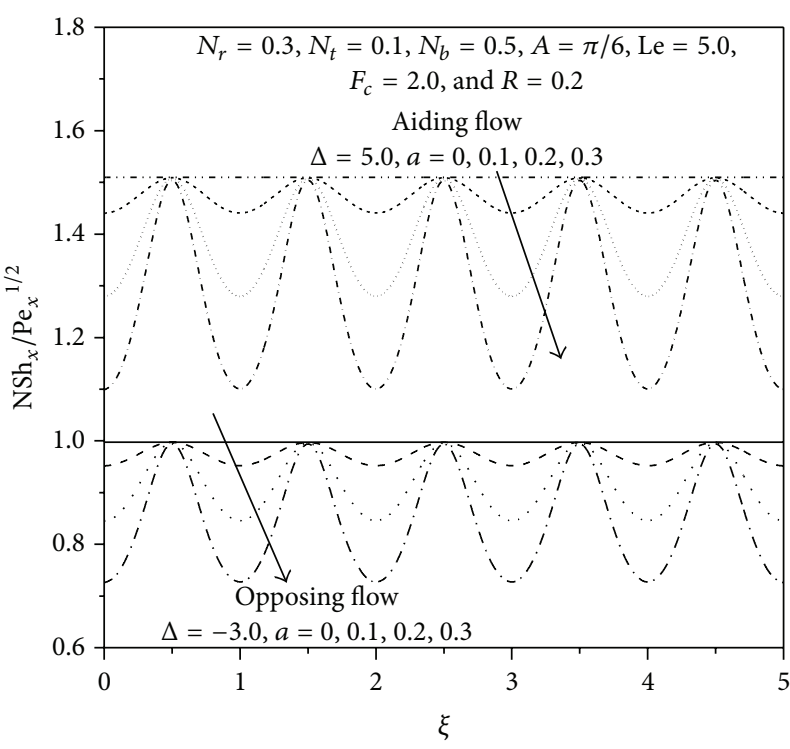

(b)

Figure 6: Effect of the wave amplitude ( $a$ ) on the heat and nanoparticle mass transfer rates.

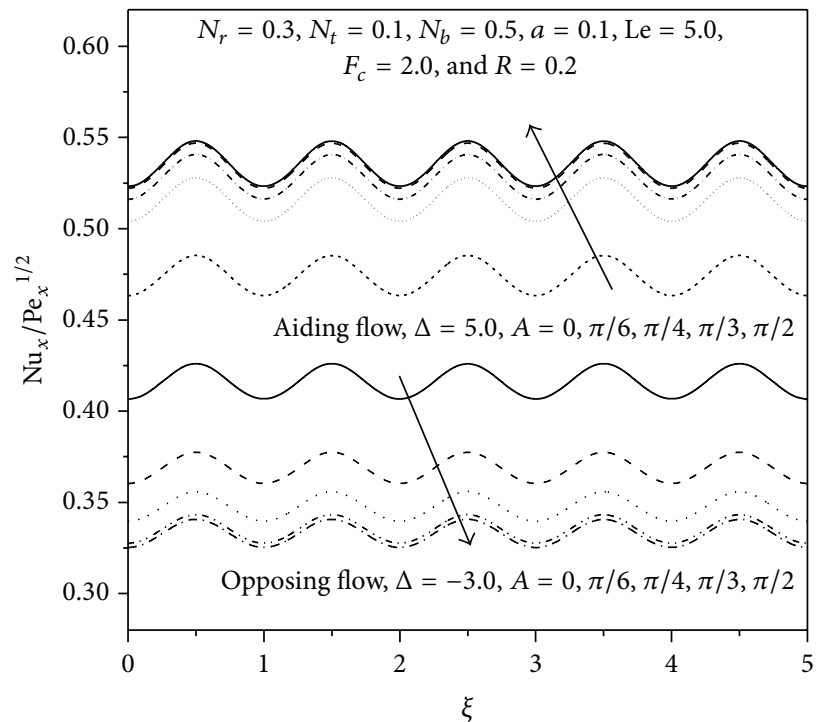

(a)

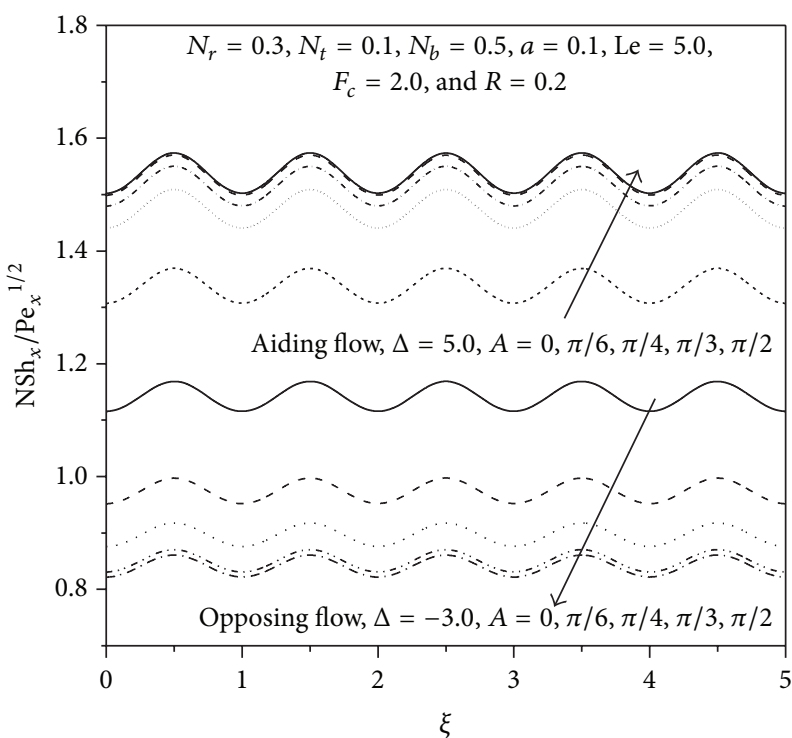

(b)

FIgURE 7: Effect of the angle of inclination $(A)$ on the heat and nanoparticle mass transfer rates.

It is revealed that the heat and nanoparticle mass transfer rate reduces with increase in the thermophoresis parameter for both aiding and opposing flows. Brownian motion is proportional to the volumetric fraction of nanoparticles in the direction from high to low concentration, whereas the thermophoresis is proportional to the temperature gradient from hot to cold. Hence, we conclude that the effect of the combination of Brownian motion and thermophoresis is to reduce the value of Nusselt number.

Figure 10 displays the effect of non-Darcy parameter $F_{c}$ on the Nusselt and nanoparticle Sherwood number. It is observed that, in the case of aiding flow, the heat and nanoparticle mass transfer reduces with increase in the value of non-Darcy parameter and a reverse trend is observed in the case of opposing flow. The effect of radiation $R$ on the heat and nanoparticle mass transfer is illustrated in Figure 11. It is noticed from Figure 11(a) that increase in the radiation parameter increases the heat transfer rates for both aiding and opposing flows. And a negligible effect of radiation on nanoparticle mass transfer is shown in Figure 11(b).

\section{Conclusions}

In this paper, the mixed convection in a nanofluid along an inclined wavy surface embedded in a non-Darcy porous medium with radiation effect is analyzed. The governing 


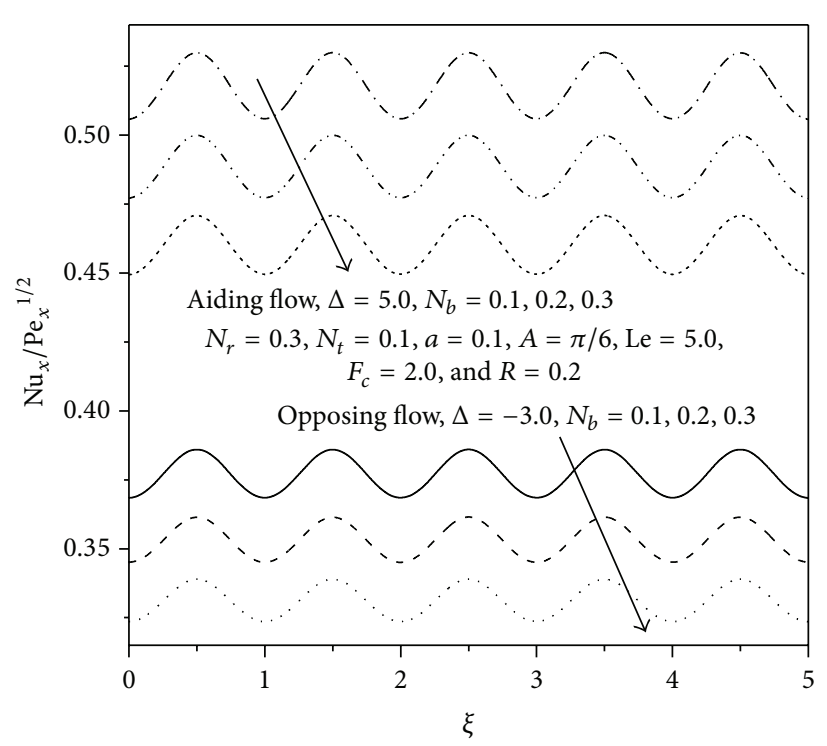

(a)

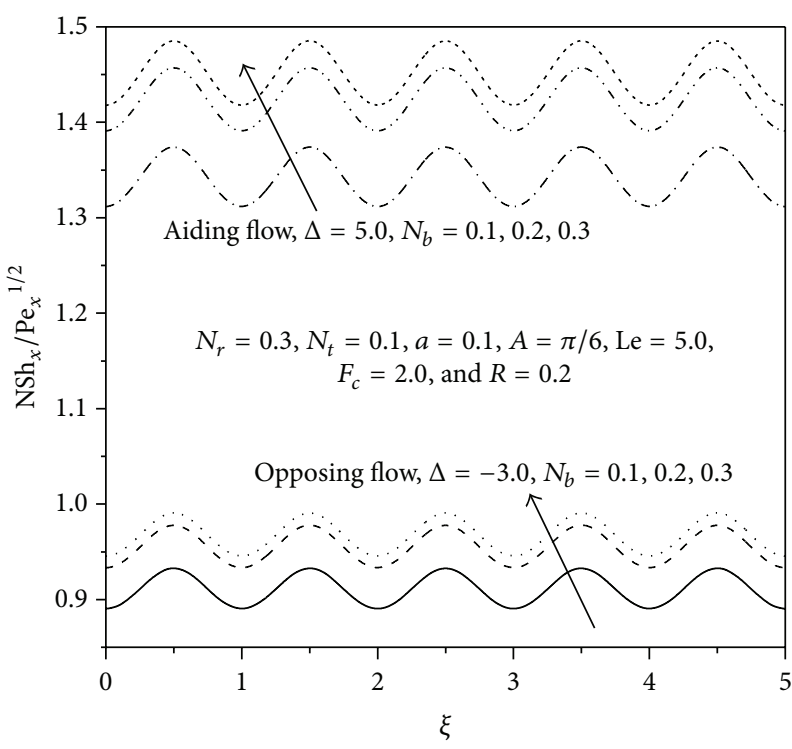

(b)

FIGURE 8: Effect of the Brownian motion parameter $\left(N_{b}\right)$ on the heat and nanoparticle mass transfer rates.

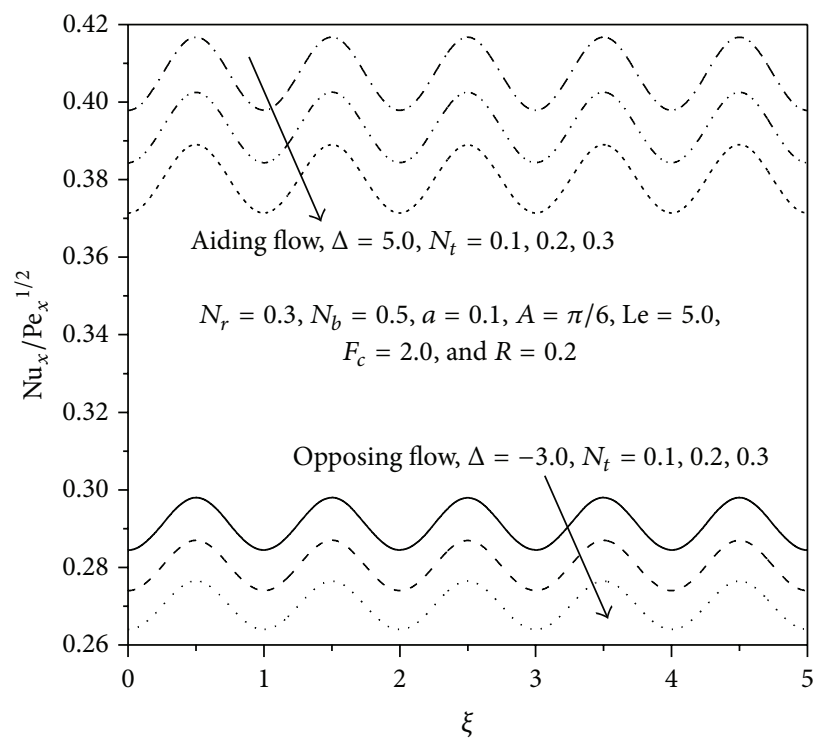

(a)

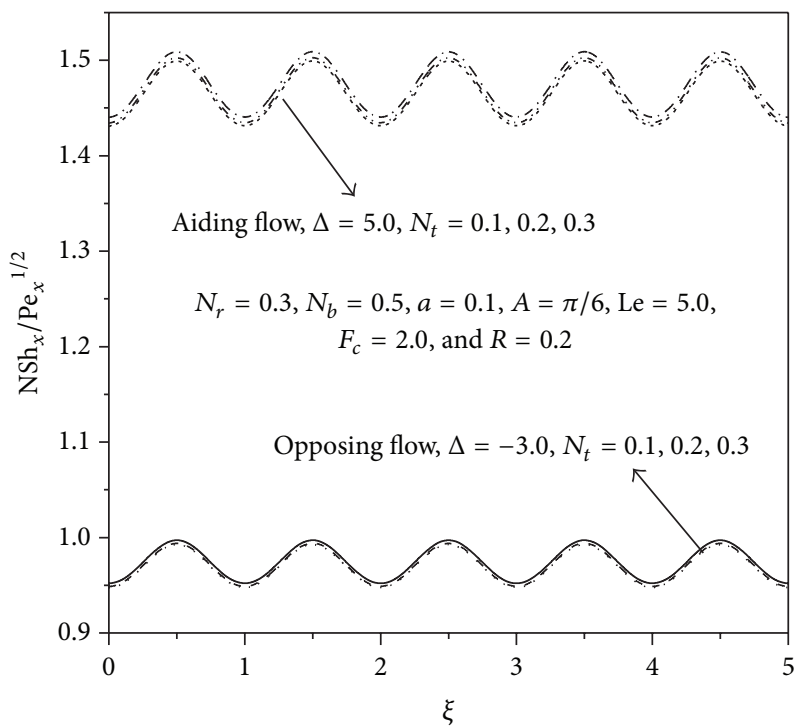

(b)

FIGURE 9: Effect of the themophoresis parameter $\left(N_{t}\right)$ on the heat and nanoparticle mass transfer rates.

equations are transformed into a set of ordinary differential equations using the appropriate similarity transformation and then are linearized using the successive linearization method. These linearized equations are solved using Chebyshev spectral collocation method. The main findings are summarized as follows:

(1) An increase in the wave amplitude enhances the velocity for aiding flow but reduces the same for opposing flow whereas the temperature and nanoparticle volume fraction reduce for aiding flow and enhances the same for opposing flow. The local heat and nanoparticle mass transfer rates are increased for both aiding and opposing flows.

(2) The effect of angle of inclination is to increase the velocity, local heat, and nanoparticle mass transfer for aiding flow and a reverse trend is observed in the case of opposing flow. Moreover, the temperature and nanoparticle volume fraction reduce for aiding flow and a reverse trend is seen for opposing flow.

(3) The effect of non-Darcy parameter is to reduce the velocity, local heat, and nanoparticle mass transfer for aiding flow and to increase in the case of opposing 


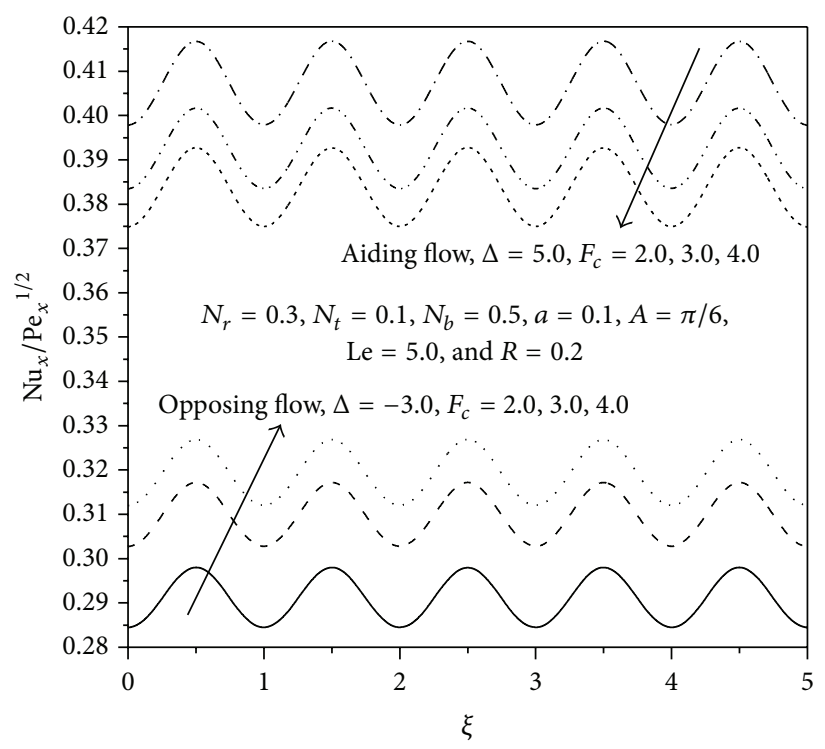

(a)

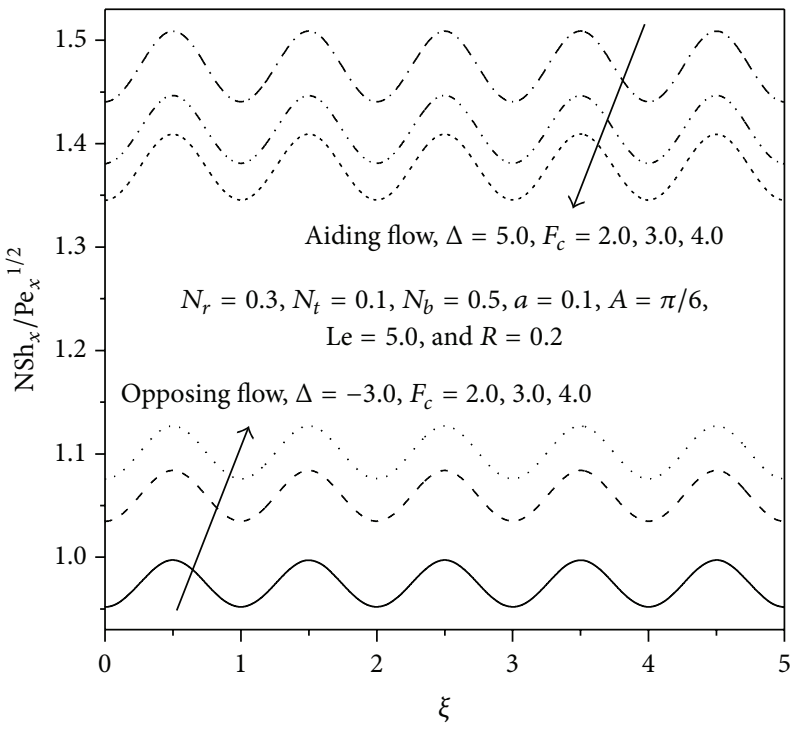

(b)

FIgURE 10: Effect of the non-Darcy parameter $\left(F_{c}\right)$ on the heat and nanoparticle mass transfer rates.

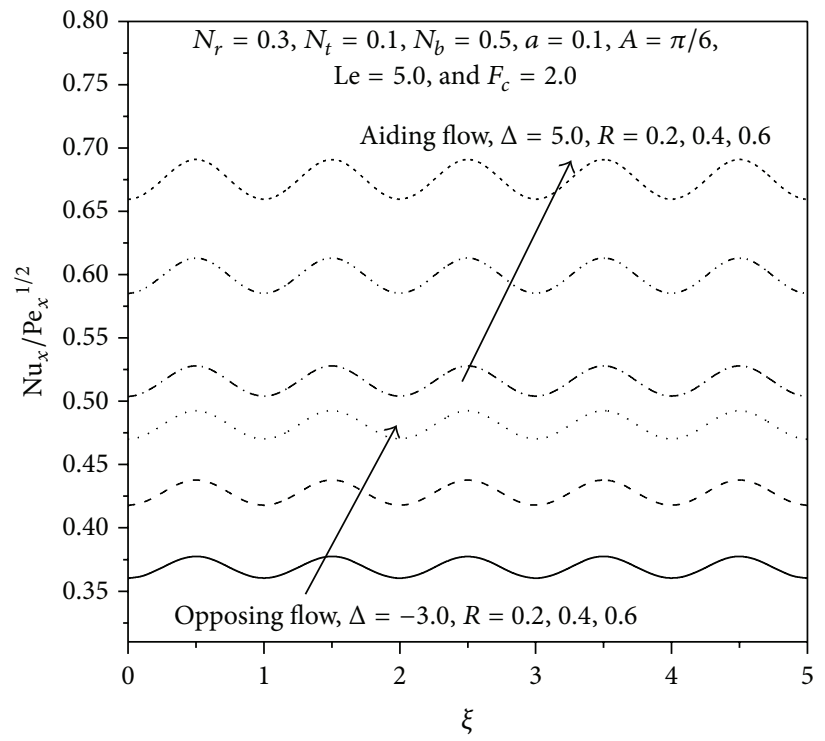

(a)

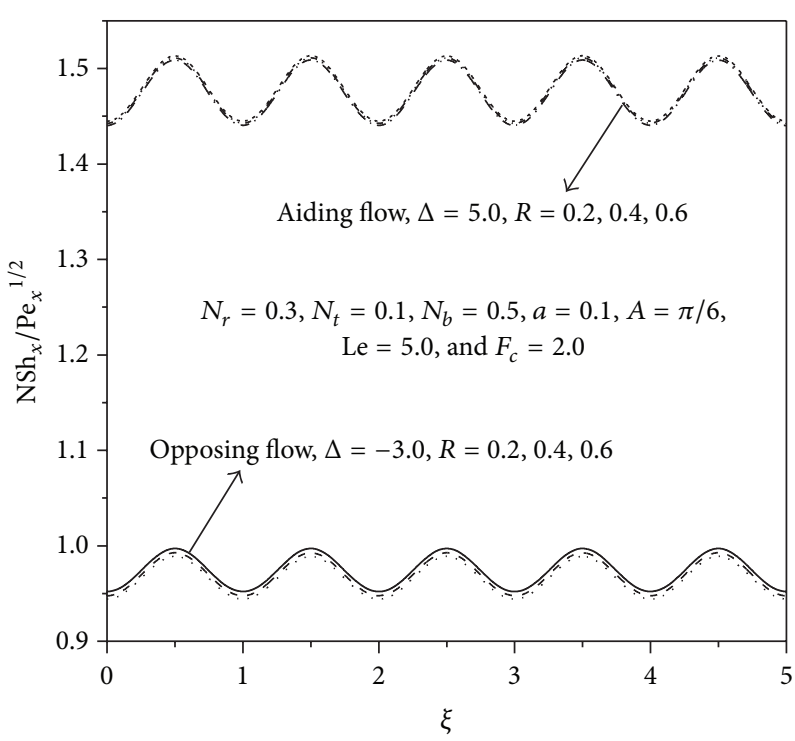

(b)

FIGURE 11: Effect of the radiation parameter $(R)$ on the heat and nanoparticle mass transfer rates.

flow while the temperature and nanoparticle volume fraction increase for aiding flow and reduce for opposing flow.

(4) The influence of radiation is to enhance the velocity and temperature for aiding flow and reduce the same in the case of opposing flow while the local heat transfer enhances for both aiding and opposing flows and a negligible effect on nanoparticle volume fraction and nanoparticle mass transfer rates is observed.

\section{Conflict of Interests}

The authors declare that there is no actual or potential conflict of interests including financial, personal, or other relationships with other people or organizations regarding the publication of this paper.

\section{References}

[1] S. U. S. Choi and J. A. Eastman, "Enhancing thermal conductivity of fluids with nanoparticles," in Developments and 
Applications of Non-Newtonian Flows, vol. 231, pp. 99-105, ASME, 1995.

[2] S. U. S. Choi, Z. G. Zhang, W. Yu, F. E. Lockwood, and E. A. Grulke, "Anomalous thermal conductivity enhancement in nanotube suspensions," Applied Physics Letters, vol. 79, no. 14, pp. 2252-2254, 2001.

[3] S. K. Das, S. U. S. Choi, W. Yu, and T. Pradeep, Nanouids: Science and Technology, Wiley-Interscience, Hoboken, NJ, USA, 2007.

[4] S. Kakaç and A. Pramuanjaroenkij, "Review of convective heat transfer enhancement with nanofluids," International Journal of Heat and Mass Transfer, vol. 52, no. 13-14, pp. 3187-3196, 2009.

[5] G. Puliti, S. Paolucci, and M. Sen, "Nanofluids and their properties," Applied Mechanics Reviews, vol. 64, no. 3, Article ID 030803, 2011.

[6] D. Wen and Y. Ding, "Effect of particle migration on heat transfer in suspensions of nanoparticles flowing through minichannels," Microfluidics and Nanofluidics, vol. 1, no. 2, pp. 183-189, 2005.

[7] S. Mirmasoumi and A. Behzadmehr, "Effect of nanoparticles mean diameter on mixed convection heat transfer of a nanofluid in a horizontal tube," International Journal of Heat and Fluid Flow, vol. 29, no. 2, pp. 557-566, 2008.

[8] K. Khanafer, K. Vafai, and M. Lightstone, "Buoyancy-driven heat transfer enhancement in a two-dimensional enclosure utilizing nanofluids," International Journal of Heat and Mass Transfer, vol. 46, no. 19, pp. 3639-3653, 2003.

[9] R. K. Tiwari and M. K. Das, "Heat transfer augmentation in a two-sided lid-driven differentially heated square cavity utilizing nanofluids," International Journal of Heat and Mass Transfer, vol. 50, no. 9-10, pp. 2002-2018, 2007.

[10] E. Abu-Nada, "Application of nanofluids for heat transfer enhancement of separated flows encountered in a backward facing step," International Journal of Heat and Fluid Flow, vol. 29, no. 1, pp. 242-249, 2008.

[11] J. Buongiorno, “Convective transport in nanofluids," Journal of Heat Transfer, vol. 128, no. 3, pp. 240-250, 2006.

[12] A. V. Kuznetsov and D. A. Nield, "Natural convective boundarylayer flow of a nanofluid past a vertical plate," International Journal of Thermal Sciences, vol. 49, no. 2, pp. 243-247, 2010.

[13] D. A. Nield and A. Bejan, Convection in Porous Media, Springer, New York, NY, USA, 4th edition, 2013.

[14] S. Ahmad and I. Pop, "Mixed convection boundary layer flow from a vertical flat plate embedded in a porous medium filled with nanofluids," International Communications in Heat and Mass Transfer, vol. 37, no. 8, pp. 987-991, 2010.

[15] A. J. Chamkha and A. M. Aly, "MHD free convection flow of a nanofluid past a vertical plate in the presence of heat generation or absorption effects," Chemical Engineering Communications, vol. 198, no. 3, pp. 425-441, 2011.

[16] A. V. Rosca, N. C. Rosca, T. Grosan, and I. Pop, "Non-Darcy mixed convection from a horizontal plate embedded in a nanofluid saturated porous media," International Communications in Heat and Mass Transfer, vol. 39, no. 8, pp. 1080-1085, 2012.

[17] P. Rana, R. Bhargava, and O. A. Bég, "Numerical solution for mixed convection boundary layer flow of a nanofluid along an inclined plate embedded in a porous medium," Computers \& Mathematics with Applications, vol. 64, no. 9, pp. 2816-2832, 2012.
[18] R. Kamali and A. R. Binesh, "Effects of nanoparticle size on nanofluids heat transfer characteristics in minichannels," Journal of Computational and Theoretical Nanoscience, vol. 10, no. 4, pp. 1027-1032, 2013.

[19] C.-K. Chen, Y.-T. Yang, and K.-H. Chang, "Entropy generation of radiation effect on laminar-mixed convection along a wavy surface," Heat and Mass Transfer, vol. 47, no. 4, pp. 385-395, 2011.

[20] A. J. Chamkha, S. Abbasbandy, A. M. Rashad, and K. Vajravelu, "Radiation effects on mixed convection over a wedge embedded in a porous medium filled with a nanofluid," Transport in Porous Media, vol. 91, no. 1, pp. 261-279, 2012.

[21] F. M. Hady, F. S. Ibrahim, S. M. Abdel-Gaied, and M. R. Eid, "Radiation effect on viscous flow of a nanofluid and heat transfer over a nonlinearly stretching sheet," Nanoscale Research Letters, vol. 7, article 229, 13 pages, 2012.

[22] G. M. Abdel-Rahman, "Thermal radiation and unsteady magnetohydrodynamic flow of nanofluid in stretching porous medium," Journal of Thermophysics and Heat Transfer, vol. 27, no. 1, pp. 142-150, 2013.

[23] A. Mahdy and S. E. Ahmed, "Laminar free convection over a vertical wavy surface embedded in a porous medium saturated with a nanofluid," Transport in Porous Media, vol. 91, no. 2, pp. 423-435, 2012.

[24] S. E. Ahmed and M. M. Abd El-Aziz, "Effect of local thermal non-equilibrium on unsteady heat transfer by natural convection of a nanofluid over a vertical wavy surface," Meccanica, vol. 48, no. 1, pp. 33-43, 2013.

[25] D. B. Ingham, J. H. Merkin, and I. Pop, "Natural convection from a semi-infinite flat plate inclined at a small angle to the horizontal in a saturated porous medium," Acta Mechanica, vol. 57, no. 3-4, pp. 183-202, 1985.

[26] C.-Y. Cheng, "Double diffusive natural convection along an inclined wavy surface in a porous medium," International Communications in Heat and Mass Transfer, vol. 37, no. 10, pp. 1471-1476, 2010.

[27] C.-Y. Cheng, "Natural convection heat transfer from an inclined wavy plate in a bidisperse porous medium," International Communications in Heat and Mass Transfer, vol. 43, pp. 69-74, 2013.

[28] E. M. Sparrow and R. D. Cess, Radiation Heat Transfer, Hemisphere, Washington, DC, USA, 1978.

[29] I. P. Warneford, Natural convection from an inclined flat plate [Ph.D. thesis], University of Nottingham, 1975.

[30] M. S. Abdallah and B. Zeghmati, "Effects of the wavy surface on free convection-radiation along an inclined plate," World Academy of Science, Engineering and Technology, vol. 43, no. 7, pp. 6-29, 2013.

[31] S. S. Motsa and S. Shateyi, "Successive linearisation solution of free convection non-Darcy flow with heat and mass transfer," in Advanced Topics in Mass Transfer, chapter 19, pp. 425-438, InTech, Rijeka, Croatia, 2006.

[32] Z. G. Makukula, P. Sibanda, and S. Motsa, "A novel numerical technique for two-dimensional laminar flow between two moving porous walls," Mathematical Problems in Engineering, vol. 2010, Article ID 528956, 15 pages, 2010.

[33] F. G. Awad, P. Sibanda, S. S. Motsa, and O. D. Makinde, "Convection from an inverted cone in a porous medium with cross-diffusion effects," Computers and Mathematics with Applications, vol. 61, no. 5, pp. 1431-1441, 2011. 
[34] C. Canuto, M. Y. Hussaini, A. Quarteroni, and T. A. Zang, Spectral Methods-Fundamentals in Single Domains, Springer, 2006.

[35] C. Ping, "Combined free and forced convection flow about inclined surfaces in porous media," International Journal of Heat and Mass Transfer, vol. 20, no. 8, pp. 807-814, 1977. 

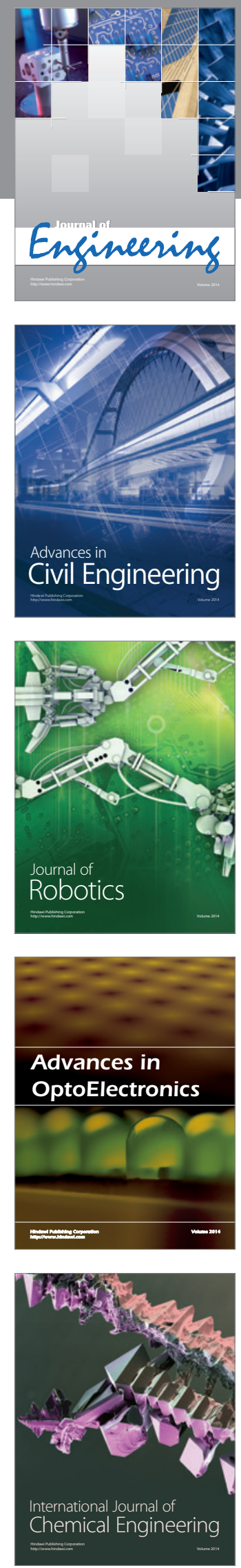

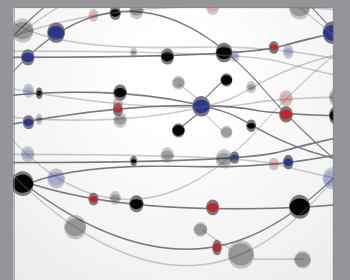

The Scientific World Journal
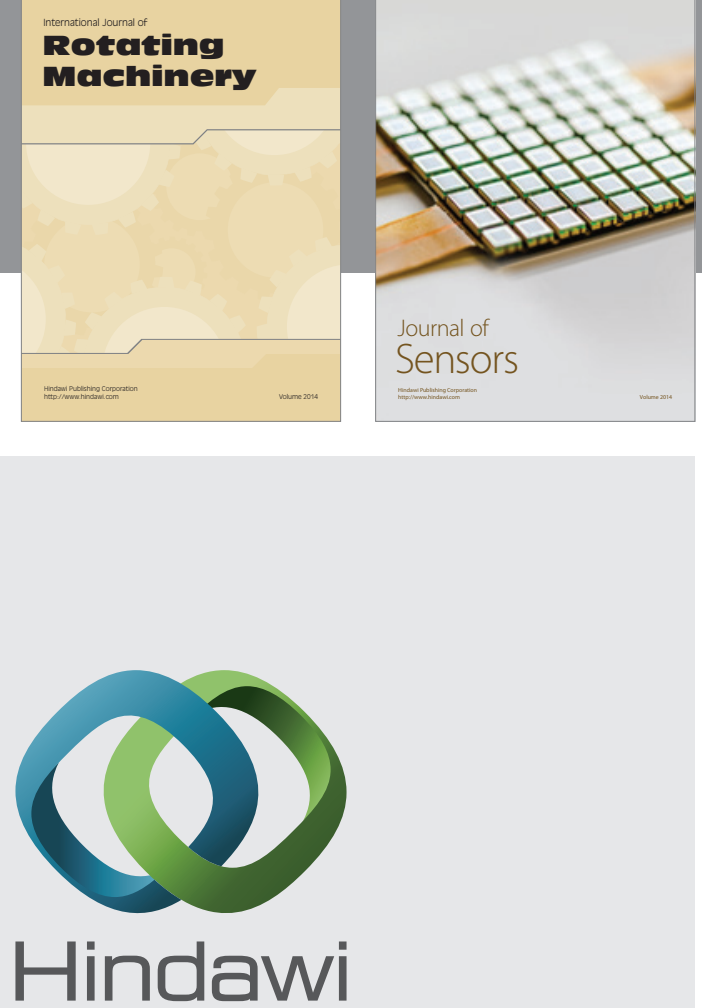

Submit your manuscripts at http://www.hindawi.com
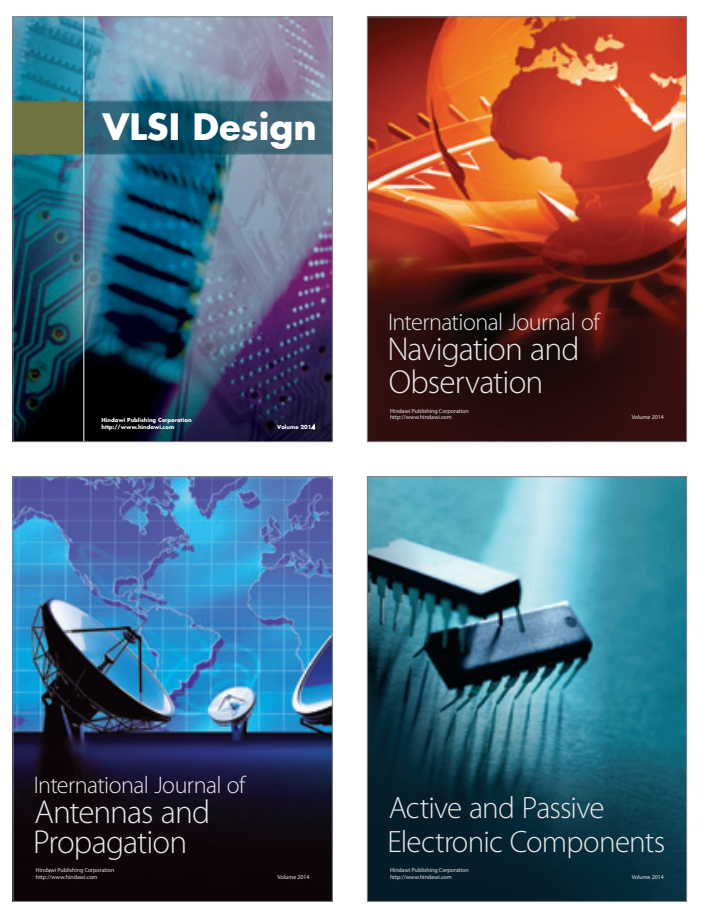
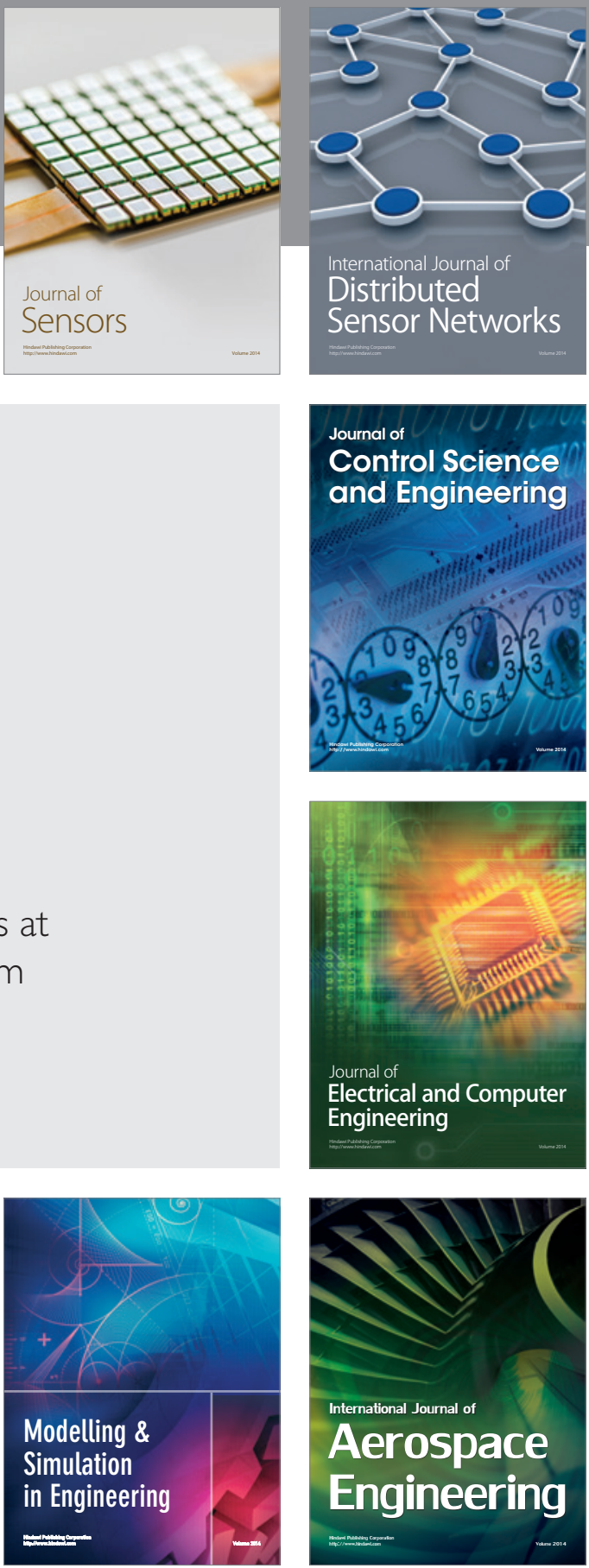

Journal of

Control Science

and Engineering
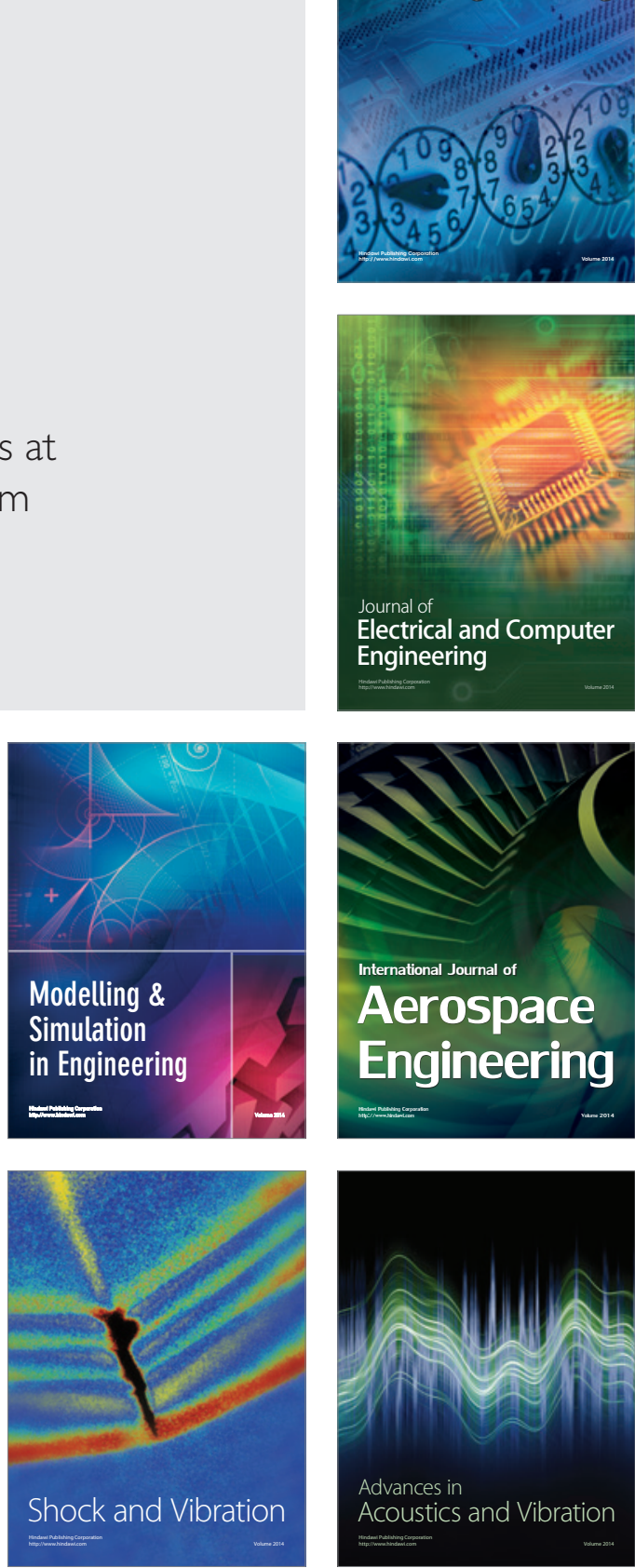